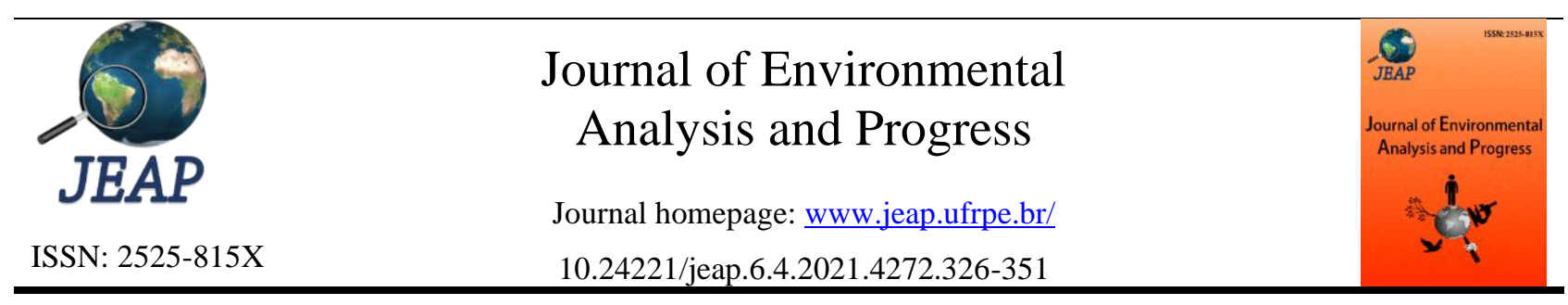

\title{
Ethnopharmacology of the angiosperms of Chapada of Araripe located in Northeast of Brazil
}

Rafael Pereira da Cruz ${ }^{\mathrm{a}}$, José Weverton Almeida-Bezerra ${ }^{\mathrm{b} *}$, Saulo Almeida de Menezes ${ }^{\mathrm{b}}$, Viviane Bezerra da Silva ${ }^{\mathrm{b}}$, Luciano Temoteo dos Santos ${ }^{\mathrm{c}}$, Maria Flaviana Bezerra Morais-Braga ${ }^{\mathrm{a}}$, José Laécio de Moraes ${ }^{\mathrm{a}}$

${ }^{\text {a }}$ Universidade Regional do Cariri-URCA. Rua Cel. Antônio Luiz, n. 1161, Pimenta, Crato-CE, Brasil. CEP: 63105-000. E-mail: rafaelcruz284@gmail.com, flavianamoraisb@yahoo.com.br, laeciomoraes.ambiental@gmail.com.

${ }^{\mathrm{b}}$ Universidade Federal de Pernambuco-UFPE. Av. Professor Moraes Rego, n. 1235, Cidade Universitária, Recife-PE, Brasil. CEP: 50670-901. E-mail: weverton.almeida@urca.br*, saulomenezes99@gmail.com, viviane.silva@urca.br.

${ }^{\mathrm{c}}$ Universidade Federal do Cariri-UFCA. Rua Icaro de Sousa Moreira, 126, Muriti, Crato-CE, Brasil. CEP: 63130-025. E-mail: luciano.temoteosantos@gmail.com.

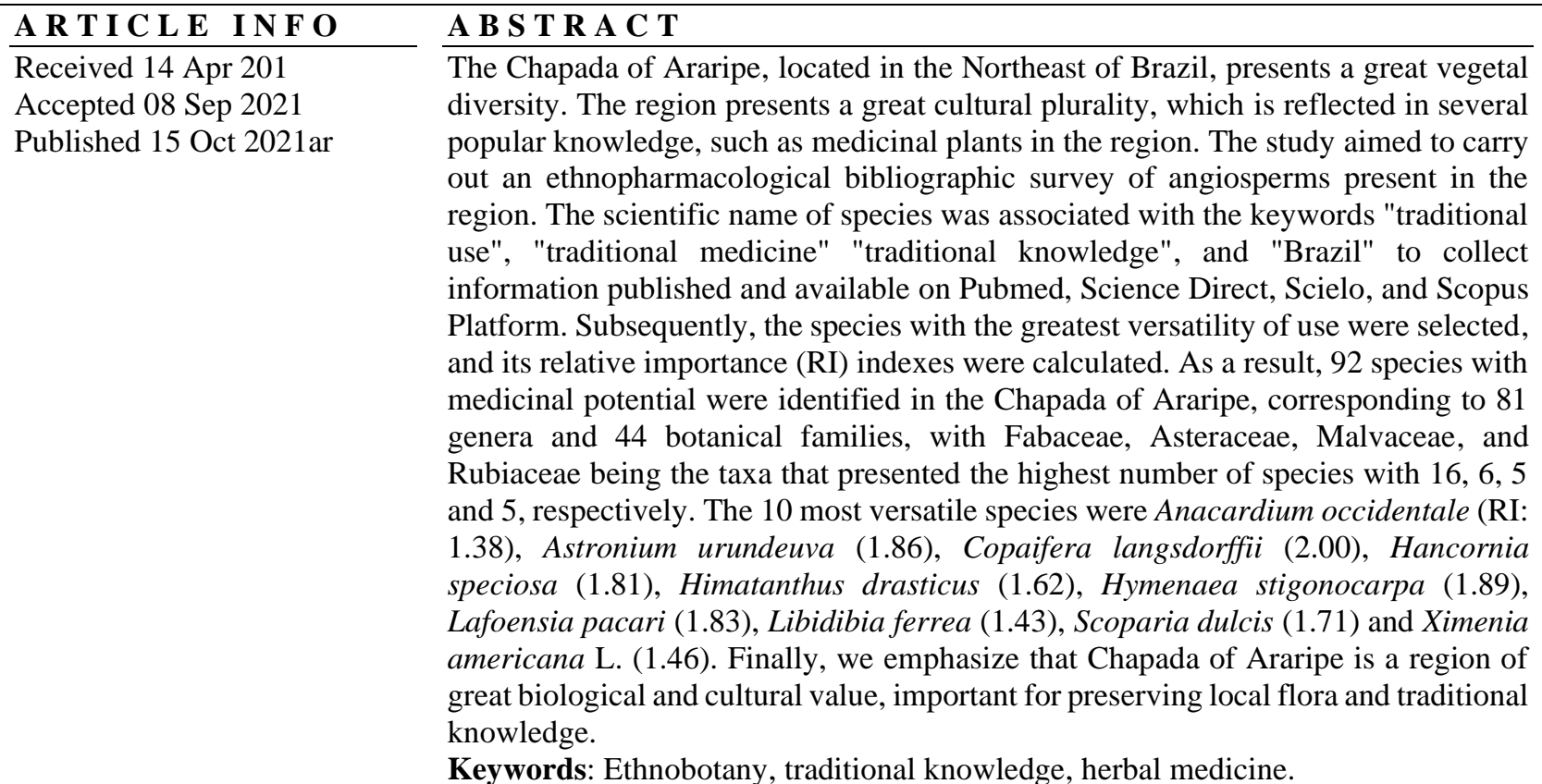

\section{Introduction}

The Brazilian flora is recognized for hosting several native species of great biological importance, responsible for approximately $20 \%$ of the planet's plant biodiversity. We can identify all this natural wealth distributed among the various phytogeographic domains in the national territory, with its characteristic and diversified vegetation (Amaral et al., 2015; Garcez et al., 2016; Macedo et al., 2018). One of these exclusively Brazilian domains is the Caatinga, a seasonally dry tropical forest located in the country's Northeast (Santos et al., 2011). There are enclaves with characteristic physiognomies in this ecosystem due to their geological formations, such as Chapada of Ibiapaba, Serra Maciço of Baturité, Serra of
Pirapora, and Chapada of Araripe (Batista et al., 2018). The latter, located in the Brazilian semi-arid region (between the states of Pernambuco, Piauí, and Ceará), is an area considered important for the protection of the Caatinga's biodiversity, being formed by two conservation units: the AraripeApodi National Forest (Flona Araripe-Apodi ) and the Chapada of Araripe Preservation Area (APAAraripe), the units are managed by environmental agencies of the public power and also by private initiative (Bastos et al., 2016; Sousa-Júnior et al., 2018; Alcântara et al., 2020). The vegetation of this region is composed of Mata Humid, Mata Seca, Caatinga, Cerrado and Cerradão (Moro et al., 2015). 
The Chapada of Araripe is among the 27 areas of Brazil of high biological value, where approximately 173 species of native plants are traditionally used by the communities that inhabit this region (IBAMA, 2004; Sousa-Júnior et al., 2018; Ribeiro et al., 2017a). However, the therapeutic potential of most of these species is still unknown. There is a need for ethnopharmacological studies to support the discovery of new herbal medicines and contribute to sustainable use practices, as the irrational exploitation of local flora can lead to the loss of native species of great biological importance (Ribeiro et al., 2014; Albuquerque et al., 2007a; Albuquerque et al., 2011).

Despite the advancement of modern medicine in recent years, the World Health Organization (WHO) recognizes that many developing countries still depend on medicinal plants for therapeutic use (WHO, 2018). Such use is mainly due to difficulties accessing health systems and services, isolation and distance from urban centers, the low economic cost concerning commercial drugs, and a culturally accepted practice (Bitu et al., 2015; Macedo et al., 2015).

In this sense, some ethnomedicinal studies provide information on the use and therapeutic potential of many plants used by traditional communities in the semi-arid Caririense (Ribeiro et al., 2014; Souza et al., 2014; Bitu et al., 2015; Macedo et al., 2015; Lemos et al., 2016), such as Astronium urundeuva (M. Allemão) Engl. (aroeira), Hancornia speciosa Gomes (mangaba), Himatanthus drasticus (Mart.) Plumel (janaguba), Caryocar coriaceum Wittm. (pequi), Dimorphandra gardneriana Tul. (faveira), Stryphnodendron rotundifolium Mart. (barbatimão) e Ximenia americana L. (ameixa). These species have great medicinal, cultural, food, and commercial value in the region, including some of which are known to have scientifically proven pharmacological and biological activities, being explored in the bioprospecting of molecules and the search for new drugs (Pereira et al., 2015; Bezerra et al., 2018; Galvão et al., 2018; Silva et al.,2018 Almeida et al., 2019).

Given the above, the objective of this study is to carry out a bibliographic survey on specialized bases of the ethnopharmacological uses of medicinal plants found in Chapada of Araripe. In addition, it was selected the most versatile species and investigating their respective pharmacological and biological activities in the literature and their main chemical classes.

\section{Material and Methods}

The study area of this research is the Chapada of Araripe (Figure 1), a region inserted in the Caatinga domain with a tabular feature of sedimentary origin, is located between the states of Pernambuco, Piaui and the extreme south of Ceará (where most of its territory is found) (Novaes \& Laurindo, 2014; Moro et al., 2015). The species documented in the Chapada of Araripe floristic inventory were consulted. This survey gathers information about the local flora collected in Herbariums and field research; in general, 474 plant species belonging to 79 families and 275 genera are listed, occurring in Chapada (Loiola et al., 2015).

The scientific name of these species was associated with the keywords "traditional use", "traditional medicine" "traditional knowledge" and "Brazil" to collect information published and available on Pubmed, Science Direct, Scielo, and Scopus platform. There was no time limit for the inclusion of publications. Scientific papers that had a research objective in the format of abstracts, monographs, dissertations, theses, book chapters, and articles with incomplete information were not considered. Before the bibliographic survey, the scientific names of the species were checked and confirmed in Flora do Brasil 2020 (http://floradobrasil.jbrj.gov.br) and The Plant List (http://www.theplantlist.org), when appropriate, species names, synonyms, and authors were corrected according to information collected on the websites. The results obtained were analyzed and summarized in a table. The species' scientific name, vernacular name, therapeutic indication, part of the plant used, forms of use, and respective citations were listed.

The relative importance index (RI) was calculated according to the methodology of Bennett \& Prance (2000) for plants that have a higher number of therapeutic indications. Relative importance is a method that quantifies the importance of a species based on its versatility, with "2" being the maximum value obtained by a species.

The calculation is made using the formula: $\mathrm{RI}=\mathrm{NBS}+\mathrm{PN}$, where RI corresponds to Relative Importance, NBS is the number of body systems of a given species (NBSS), divided by the total number of body systems assigned to the most versatile species (NBSVS); that is, NBS= NBSS/NBSVS, while PN corresponds to the number of properties attributed to a given species (NPS), divided by the total number of properties attributed to the most versatile species (NPVS), equivalent to PN = NPS/NPVS (Silva et al ., 2010). The calculation of this index was based on the 
distribution of the therapeutic indications of the most versatile species in body systems, according to the International Statistical Classification of
Diseases and Related Health Problems (ICD-10) (WHO, 2019).

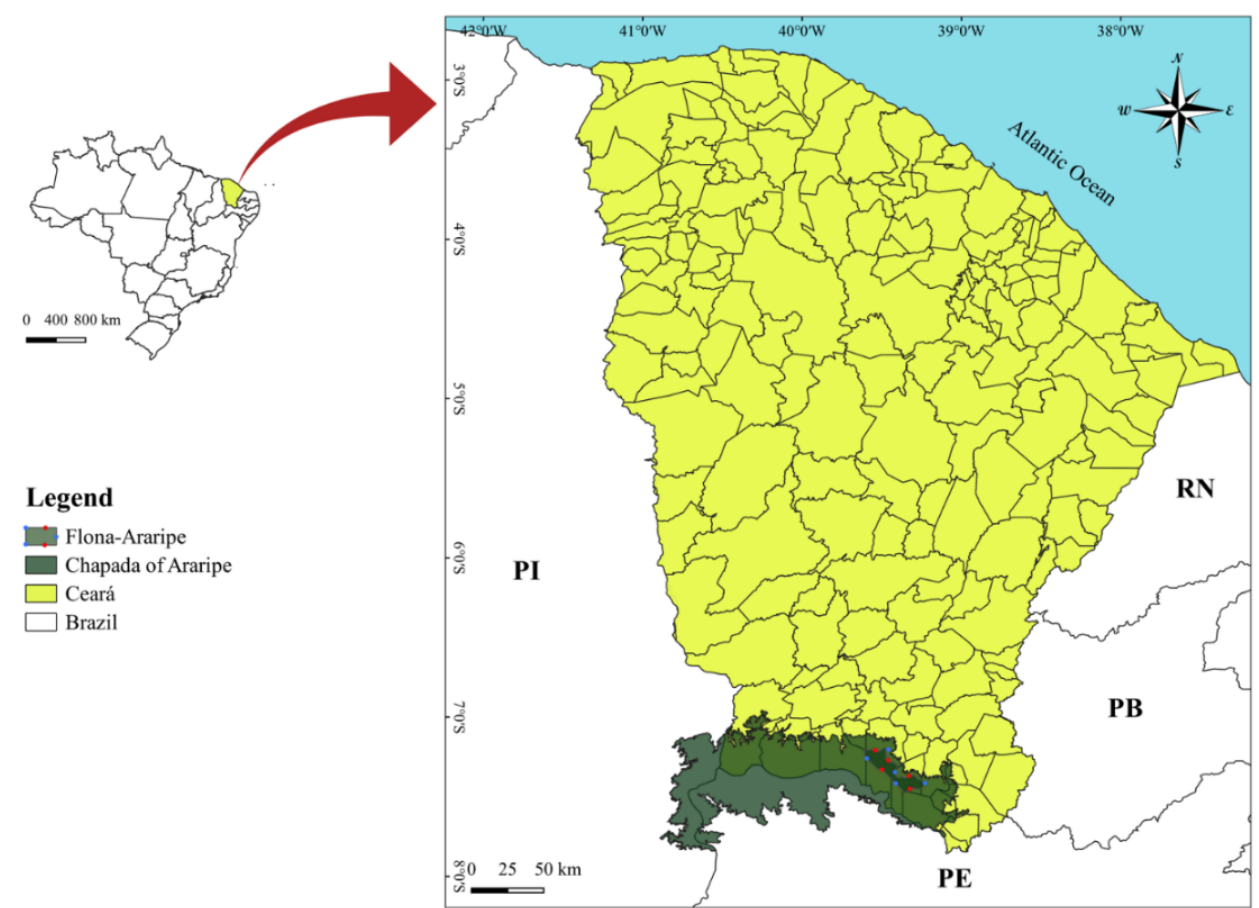

Figure 1. Geographic location of the study area in Chapada of Araripe, Northeast, Brazil Font: Cruz, R. P. (2021).

\section{Results and Discussion}

Diversity of medicinal species

In this study, a total of 92 species found in Chapada of Araripe were found, which are used in traditional medicine, corresponding to 81 genera and 44 families (Table 1), with Fabaceae, Asteraceae, Malvaceae, and Rubiaceae being the taxa that presented the highest number of species with $16,6,5$ and 5, respectively. The versatility of Fabaceae is also cited in other ethnopharmacological studies in cerrado areas of the Chapada of Araripe, considered the family with a greater abundance of species used in traditional medicine in this region (Ribeiro et al., 2014; Macedo et al., 2015; Macedo et al., 2016).

Most of the listed species are trees (41\%), followed by shrubs $(28 \%)$ and terrestrial herbs $(16 \%)$, as shown in Figure 2. Some research indicates a relationship between the form of growth and the presence of chemical compounds, being that trees generally have higher amounts of phenols, tannins, alkaloids, triterpenes, and quinones when compared to shrubs and herbaceous species; these classes have pharmacological and biological actions (Almeida et al., 2005; Alencar et al., 2010).

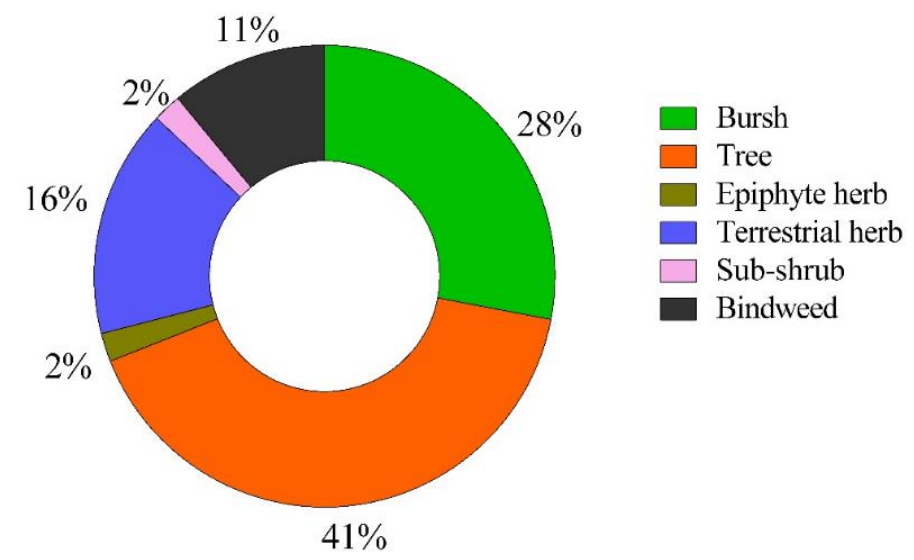

Figure 2. Percentage distribution of the form of growth of medicinal plants found in Chapada of Araripe, Northeast Brazil. Font: Cruz, R. P. (2021). 
The most used vegetable parts were barks (26\%), leaves (25\%), and roots (15\%) (Figure 3). Also less frequently used were fruits, flowers, stems, seeds, and plant products, such as sap, latex, and resin. Ribeiro et al. (2017a) stated that the disordered collection of bark, stem, roots, resin, and latex can cause irreversible damage to the plant, decreasing the density and richness of species in ecosystems. It is especially aggravated when the species has several therapeutic indications or when using the entire plant (5\%), as in the case of Scoparia dulcis and Ageratum conyzoides, being necessary to reinforce the importance of sustainable use for the conservation of priority native species.

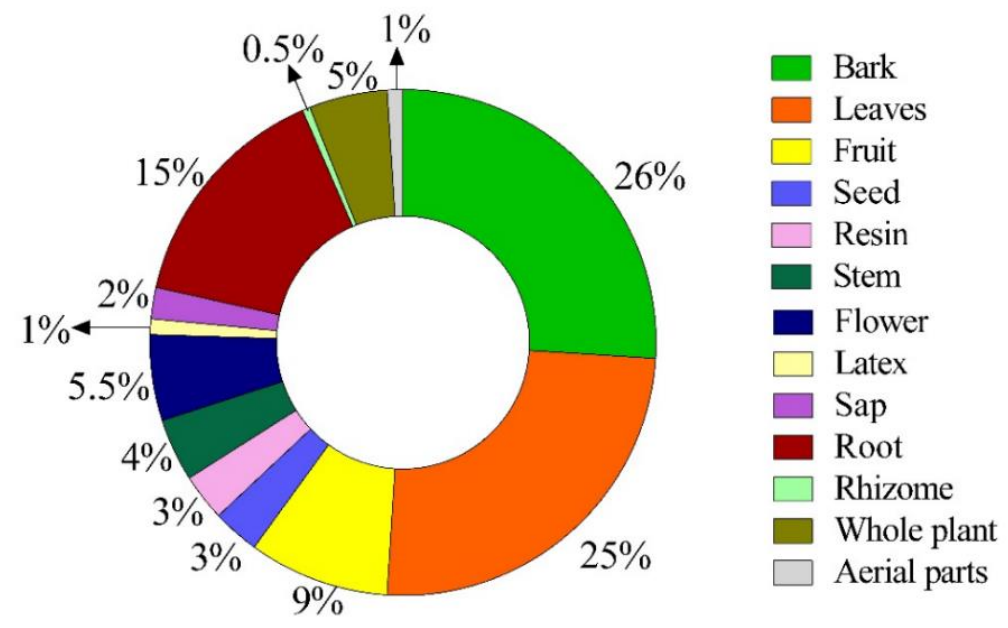

Figure 3. Percentage of the main parts used in the ethnopharmacological preparation of angiosperms from Chapada of Araripe, Northeastern Brazil. Font: Cruz, R. P. (2021).

It was identified 11 forms of preparation, the most prevalent being decoction (26\%), followed by maceration (24\%) and infusion (23\%), as shown in Figure 4. Some specificities in the preparation method were identified, as in the case of the species $H$. drasticus, where latex with water is indicated to treat various diseases. This mixture is popularly known in the region of Cariri Cearense as "leite-da-janaguba". Due to the extraction of this medicinal plant for the sale of latex, the species suffered high anthropogenic pressure, and there is currently specific legislation for its management (Badaulf \& Santos, 2013).
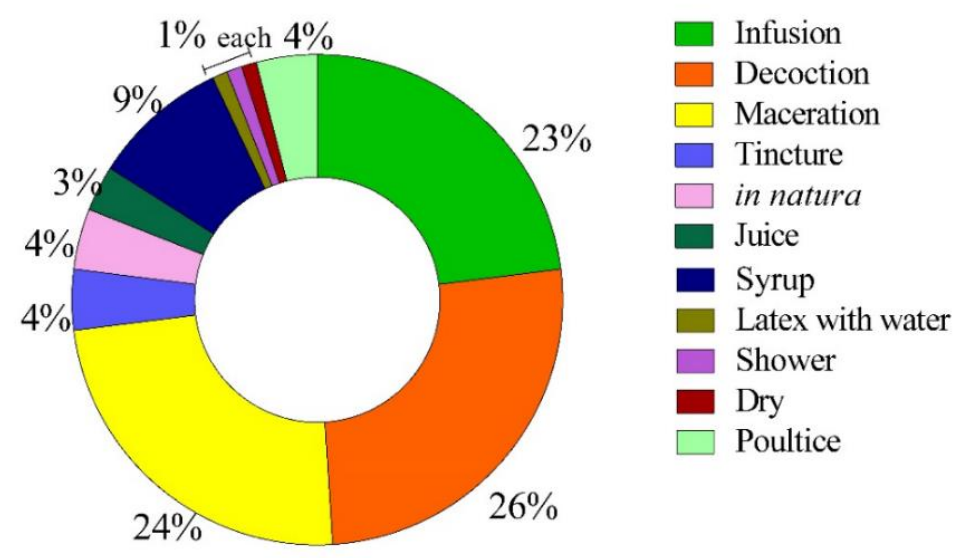

Figure 4. Percentage of the preparation methods of medicinal plants found in the Chapada of Araripe, Northeast Brazil. Font: Cruz, R. P. (2021).

Medicinal species with great versatility of use

Regarding the number of therapeutic indications, Copaifera langsdorffii was the species that showed greater versatility, being cited for the treatment of 38 diseases, followed by Lafoensia pacari (35 therapeutic indications), Hymenaea stigonocarpa (36), A. urundeuva (33), H. speciosa (31), S. dulcis (28), H. drasticus, X. americana (both with 27 indications), Libidibia ferrea (21) and Anacardium occidentale (21) (Table 1) (Figure $5)$. 
Table 1. List of species of medicinal use of Chapada of Araripe, Northeastern Brazil. Habit: Tree (Tr), Shrubby (Sh), Shrublet ( $\mathrm{Sl}$ ), Climbing plants (Cp), Herbaceous (He), Epiphyte (Ep); Part used: Stem (St), Stem bark (Sb), Leaf (Le), Flower (Fl), Fruit (Fr), Latex (La), Sap (Sa), Seed (Se), Root (Ro), Resin (Re), Rhizomet (Rh), Areas parts (Ap), Whole plant (Wp). * Brazilian distilled alcoholic beverage. Font: Cruz, R. P. (2021).

\begin{tabular}{|c|c|c|c|c|c|c|}
\hline Família/Espécie & Vernacular name & Habit & Therapeutic indication & $\begin{array}{l}\text { Part } \\
\text { used }\end{array}$ & Preparation & References \\
\hline \multicolumn{7}{|l|}{ AMARANTHACEAE } \\
\hline $\begin{array}{l}\text { Alternanthera brasiliana (L.) } \\
\text { Kuntz }\end{array}$ & $\begin{array}{l}\text { quebra-panela, } \\
\text { acônito, ervanço, } \\
\text { terramicina, } \\
\text { benzetacil }\end{array}$ & $\mathrm{He}$ & $\begin{array}{l}\text { Influenza, common cold, healing, cancer, } \\
\text { diarrhea, headache, uterine inflammation, } \\
\text { vaginal discharge, postoperative infection, } \\
\text { throat inflammation, inflammations in } \\
\text { general, localized pain, fever, worms, } \\
\text { digestive problems, diuretic, expectorant }\end{array}$ & $\begin{array}{l}\text { Fl, } \\
\text { Ro }\end{array}$ & $\begin{array}{l}\text { Infusion, } \\
\text { decoction }\end{array}$ & $\begin{array}{c}\text { Agra et al. (2007); } \\
\text { Albuquerque et al. (2007a); } \\
\text { Bieski et al. (2015); Lemos et } \\
\text { al. (2016); Miguéis et al. } \\
\text { (2019); Yazbek et al. (2019) }\end{array}$ \\
\hline Alternanthera tenella Colla & $\begin{array}{l}\text { carrapichinho, } \\
\text { corrente, quebra- } \\
\text { panela, anador }\end{array}$ & $\mathrm{He}$ & $\begin{array}{c}\text { Influenza, fever, headache, antiseptic urinary } \\
\text { tract, diuretic }\end{array}$ & Le & $\begin{array}{l}\text { Infusion, } \\
\text { decoction }\end{array}$ & $\begin{array}{c}\text { Dorigoni et al. (2001); Agra et } \\
\text { al. (2007); Albuquerque et al. } \\
\text { (2007a) }\end{array}$ \\
\hline \multicolumn{7}{|l|}{ ANACARDIACEAE } \\
\hline Anacardium occidentale L. & cajuí, cajueiro, caju & $\operatorname{Tr}$ & $\begin{array}{l}\text { Urinary retention, edema, inflammations in } \\
\text { general, healing, constipation, rheumatism, } \\
\text { diabetes, bleeding, mycoses, diarrhea, } \\
\text { gastritis, infections in general, tooth } \\
\text { inflammation, toothache, dermatitis, } \\
\text { pneumonia, tuberculosis, blows, antiseptic, } \\
\text { uterine inflammation, hepatitis, gingivitis, } \\
\text { kidney infection, vaginal discharge, magical- } \\
\text { religious use }\end{array}$ & $\begin{array}{l}\mathrm{Sb}, \\
\text { Le, } \\
\text { Ro, } \\
\text { Fr }\end{array}$ & $\begin{array}{l}\text { Decoction, } \\
\text { infusion, } \\
\text { maceration }\end{array}$ & $\begin{array}{l}\text { Albuquerque et al. (2007a); } \\
\text { Albuquerque et al. (2007b) } \\
\text { Santos et al. (2009); Ribeiro } \\
\text { et al. (2014); Bitu et al. } \\
\text { (2015); Saraiva et al. (2015); } \\
\text { Vieira et al. (2015); Ribeiro et } \\
\text { al. (2017b); Mesquita \& } \\
\text { Tavares-Martins (2018) }\end{array}$ \\
\hline Astronium fraxinifolium Schott. & $\begin{array}{l}\text { gonçalave, gonçalo- } \\
\text { alves, aroeirinha }\end{array}$ & $\operatorname{Tr}$ & $\begin{array}{l}\text { Cough, influenza, back pain, fever, } \\
\text { expectorant, AIDS }\end{array}$ & $\begin{array}{l}\mathrm{Sb} \\
\text { Ro }\end{array}$ & $\begin{array}{l}\text { Decoction, } \\
\text { Infusion, } \\
\text { maceration }\end{array}$ & $\begin{array}{l}\text { Ribeiro et al. (2014); Macedo } \\
\text { et al. (2015); Ribeiro et al. } \\
\text { (2017b) }\end{array}$ \\
\hline $\begin{array}{l}\text { Astronium urundeuva (M. } \\
\text { Allemão) Engl. }\end{array}$ & $\begin{array}{l}\text { aroeira, aroeira-do- } \\
\text { sertão }\end{array}$ & $\operatorname{Tr}$ & $\begin{array}{l}\text { Inflammations in general, cough, pains in } \\
\text { general, allergy, healing, bronchitis, } \\
\text { menstrual colic, cystitis, urethritis, diarrhea, } \\
\text { acne, ulcer, bone fracture, muscle cramp, } \\
\text { cancer, rheumatism, vaginal discharge, } \\
\text { asthma, influenza, itching, tuberculosis, } \\
\text { gastritis, gonorrhea, gingivitis, anemia, } \\
\text { diphtheria, arthritis, insect bites, bacterial } \\
\text { infection, mycoses, irregular menstruation, } \\
\text { bleeding, rheumatic fever, magical-religious } \\
\text { use. }\end{array}$ & $\begin{array}{l}\mathrm{Sb} \\
\mathrm{St}, \\
\mathrm{Le} \\
\mathrm{Ro} \\
\mathrm{Re}\end{array}$ & $\begin{array}{l}\text { Decoction, } \\
\text { infusion, } \\
\text { maceration, } \\
\text { poultice, sirup }\end{array}$ & $\begin{array}{l}\text { Agra et al. (2007); } \\
\text { Albuquerque et al. (2007a); } \\
\text { Albuquerque et al. (2007b); } \\
\text { Albuquerque \& Oliveira } \\
\text { (2007); Ribeiro et al. (2014); } \\
\text { Pereira-Júnior et al. (2014); } \\
\text { Bitu et al. (2015); Saraiva et } \\
\text { al. (2015); Penido et al. } \\
\text { (2016); Ribeiro et al. (2017b) }\end{array}$ \\
\hline
\end{tabular}

\section{ANNONACEAE}




\begin{tabular}{|c|c|c|c|c|c|c|}
\hline Annona coriacea Mart. & $\begin{array}{l}\text { ariticu, araticum, } \\
\text { pinha, fruto-da- } \\
\text { quaresma }\end{array}$ & Sh & $\begin{array}{l}\text { Snakebite, thrombosis, dermatitis, depurative, } \\
\text { hypertension }\end{array}$ & $\begin{array}{l}\text { Le, } \\
\mathrm{Sb}, \mathrm{Fr}\end{array}$ & $\begin{array}{l}\text { Maceration, } \\
\text { decoction }\end{array}$ & $\begin{array}{l}\text { Ribeiro et al. (2014); Souza et } \\
\text { al. (2014); Macedo et al. } \\
\text { (2015); Ribeiro et al. (2017b) }\end{array}$ \\
\hline $\begin{array}{l}\text { Duguetia furfuracea (A.St.- } \\
\text { Hil.) Saff. }\end{array}$ & $\begin{array}{l}\text { pinha-braba, ata- } \\
\text { brava, pariri, } \\
\text { araticum-seco }\end{array}$ & Sh & $\begin{array}{l}\text { Vaginal discharge, cancer, anemia, } \\
\text { hypertension, diuretic, kidney infection, bone } \\
\text { fracture, rheumatism }\end{array}$ & $\begin{array}{l}\text { Le, } \\
\text { Ap, } \\
\text { Ro }\end{array}$ & $\begin{array}{l}\text { Decoction, } \\
\text { infusion, } \\
\text { maceration, sirup }\end{array}$ & $\begin{array}{l}\text { Rodrigues e Carvalho (2001); } \\
\text { Ribeiro et al. (2017b) }\end{array}$ \\
\hline
\end{tabular}

\section{APOCYNACEAE}

Hancornia speciosa Gomes

mangaba, mangavamansa, mangabeira

$\operatorname{Tr}$

Blows, uterine inflammation, stomach pain, gastritis, varicose veins, hernia (abdominal),

ulcer, inflammations in general, cancer,

uterine myoma, dermatitis, infections in

general, diarrhea, dysentery, furuncle, urinary infection, diabetes, hypertension,

labyrinthitis, eye irritation, indigestion, back pain, anomaly of the female reproductive system, menopause disorders, thyroid,

healing, bone fracture, hematoma, worms, rehydration

Ulcer, cancer, healing, worms, inflammations

in general, rheumatism, hemorrhoids, erectile

Himatanthus drasticus (Mart.)

Plumel

Secondatia floribunda A.DC. $\begin{aligned} & \text { catuaba-preta, } \\ & \text { catuaba-de-cipó } \\ & \text { catuaba-de-ram }\end{aligned}$

\section{ARALIACEAE}

Schefflera morototoni (Aubl.)

Maguire, Steyerm. \& Frodin.

var. morototoni

\section{ASTERACEAE}

Acanthospermum australe

(Loefl.) Kuntze

Sh Aphrodisiac, sexual impotence, internal infections, antiseptic, diarrhea, fever, tonic, dysfunction, uterine myoma, mycoses,

gallbladder problems, arthritis, laxative,

janaguba

$\operatorname{Tr}$

catuaba-preta,

tacapemba, cincodedos carrapicho, carrapicho-de-ovelha, carrapicho-decarneiro h, gastritis, diabetes, inflammation of the

liver, hernia (abdominal), uterine inflammation, throat inflammation, diarrhea, indigestion, influenza, stomach pain, anemia, varicose veins, thyroid inflammation

\section{Fl, Decoction, \\ infusion,}

maceration, latex

with water
Ribeiro et al. (2014); Soares et al. (2014), Souza et al.

(2014); Bitu et al. (2015);

Macedo et al. (2015); Vieira et al. (2015)

Infusion,

Le, maceration, in

Ribeiro et al. (2014); Souza et al. (2014); Macedo et al., 'cachaça'*

Infections in general, bleeding, post-

infarction, inflammations in general, rheumatism, pains in general

\section{Ro Maceration}

Ribeiro et al. (2017b)
Bronchitis, influenza, diuretic, intestinal worms, inflammations in general
Se,

Ro,

$\mathrm{Wp}$
Decoction, infusion, maceration
Rodrigues \& Carvalho (2001); Souza \& Felfili (2006);

Ustulin et al. (2009); Ribeiro et al. (2017b) 


\begin{tabular}{|c|c|c|c|c|c|c|}
\hline $\begin{array}{l}\text { Achyrocline satureioides (Lam.) } \\
\text { DC. }\end{array}$ & $\begin{array}{l}\text { macela-do-campo, } \\
\text { carrapicho-de-agulha, } \\
\text { vareda }\end{array}$ & $\mathrm{He}$ & $\begin{array}{l}\text { Throat inflammation, fever, indigestion, } \\
\text { stomach pain, malaria, asthma, bronchitis, } \\
\text { influenza, sinusitis, pains in the liver, pain } \\
\text { intestine, headache }\end{array}$ & Le & $\begin{array}{l}\text { Infusion, } \\
\text { decoction }\end{array}$ & $\begin{array}{l}\text { Negrelle and Fornazzari } \\
\text { (2007); Oliveira et al. (2012); } \\
\text { Lemos et al. (2016); Ribeiro } \\
\text { et al. (2017b) }\end{array}$ \\
\hline Ageratum conyzoides $\mathrm{L}$. & $\begin{array}{l}\text { mentrasto, erva-de- } \\
\text { são-joão }\end{array}$ & $\mathrm{He}$ & $\begin{array}{l}\text { Diarrhea with colic, infections in general, } \\
\text { common cold, postpartum recovery, asthma, } \\
\text { spasms, bronchitis, hematoma, skin allergy, } \\
\text { pneumonia, stomach pain, inflammations in } \\
\text { general, inflammation injury, menstrual colic, } \\
\text { expectorant, irregular menstruation }\end{array}$ & $\begin{array}{l}\text { Ro, } \\
\text { Le, } \\
\text { Wp, } \\
\text { Sb }\end{array}$ & $\begin{array}{l}\text { Decoction, } \\
\text { infusion, juice, } \\
\text { sirup, maceration }\end{array}$ & $\begin{array}{l}\text { Rodrigues \& Carvalho (2001); } \\
\text { Silva et al. (2005); Ribeiro et } \\
\text { al. (2017b); Yazbek et al. } \\
\text { (2019) }\end{array}$ \\
\hline Bidens bipinnata $\mathrm{L}$. & $\begin{array}{l}\text { Espinho-de-agulha, } \\
\text { carrapicho }\end{array}$ & $\mathrm{He}$ & Worms & Ro & Decoction & Silva et al. (2014a) \\
\hline $\begin{array}{l}\text { Conocliniopsis prasiifolia (DC.) } \\
\text { R.M.King \& H.Rob. }\end{array}$ & aleluia & $\mathrm{He}$ & Influenza, common colds & Ap & Infusion, sirup & Agra et al. (2007) \\
\hline $\begin{array}{l}\text { Emilia sonchifolia (L.) DC. ex } \\
\text { Wight }\end{array}$ & serralha & $\mathrm{He}$ & Gastritis & Le & Decoction & Yazbek et al. (2019) \\
\hline \multicolumn{7}{|l|}{ BIGNONIACEAE } \\
\hline $\begin{array}{l}\text { Fridericia chica (Bonpl.) } \\
\text { L.G.Lohmann }\end{array}$ & crajiru & $\mathrm{Cp}$ & Malaria & Le & Infusion & Frausin et al. (2015) \\
\hline $\begin{array}{l}\text { Pyrostegia venusta } \\
\text { (Ker Gawl.) Miers }\end{array}$ & $\begin{array}{l}\text { cipó-de-São- } \\
\text { João, unha-de- } \\
\text { lagartixa }\end{array}$ & $\mathrm{Cp}$ & Gastritis, vitiligo, kidney pain & $\begin{array}{l}\text { Le, } \\
\text { Rh }\end{array}$ & Maceration & Ribeiro et al. (2017b) \\
\hline \multicolumn{7}{|l|}{ BORAGINACEAE } \\
\hline Cordia rufescens A.DC. & $\begin{array}{l}\text { grão-de-galo, uva- } \\
\text { brava }\end{array}$ & Sh & Earache & $\mathrm{Fr}$ & Maceration & Ribeiro et al. (2014) \\
\hline Varronia curassavica Jacq. & erva-baleeira & Sh & Hematoma & Le & Maceration & Yazbek et al. (2019) \\
\hline \multicolumn{7}{|l|}{ BROMELIACEAE } \\
\hline Tillandsia recurvata $(\mathrm{L}.) \mathrm{L}$. & barba-de-velho & Ep & Rheumatism, ulcer, hemorrhoids & Wp & Decoction & Agra et al. (2007) \\
\hline Tillandsia streptocarpa Baker & gravatá-do-ar & Ep & Laxative, vomit & $\mathrm{Wp}$ & Decoction & Agra et al. (2007) \\
\hline \multicolumn{7}{|l|}{ BURSERACEAE } \\
\hline $\begin{array}{l}\text { Protium heptaphyllum (Aubl.) } \\
\text { Marchand subsp. heptaphyllum }\end{array}$ & $\begin{array}{l}\text { corcunda, amescla, } \\
\text { almescla, alméceha, } \\
\text { breu-amarelo, breu- } \\
\text { preto }\end{array}$ & $\operatorname{Tr}$ & $\begin{array}{l}\text { American trypanosomiasis, bleeding, anxiety, } \\
\text { bronchitis, sinusitis, indigestion, stroke, } \\
\text { respiratory disorders, healing, headache }\end{array}$ & $\begin{array}{l}\mathrm{Le}, \\
\mathrm{Fr} \\
\mathrm{Sb} \\
\mathrm{Re}\end{array}$ & $\begin{array}{l}\text { Decoction, } \\
\text { maceration, sirup }\end{array}$ & $\begin{array}{l}\text { Ribeiro et al. (2017b); Pagini } \\
\text { et al. (2017) }\end{array}$ \\
\hline \multicolumn{7}{|l|}{ CARYOCARACEAE } \\
\hline Caryocar coriaceum Wittm. & $\begin{array}{l}\text { Pequi, piqui, } \\
\text { pequizeiro }\end{array}$ & $\operatorname{Tr}$ & $\begin{array}{l}\text { Throat inflammation, bronchitis, cough, } \\
\text { asthma, influenza, rheumatism, furuncle, } \\
\text { swelling, throat inflammation, blows, fever, } \\
\text { burns, pains in general, indigestion, } \\
\text { expectorante }\end{array}$ & $\mathrm{Fr}, \mathrm{Fl}$ & Sirup, decoction & $\begin{array}{l}\text { Ribeiro et al. (2014); Macedo } \\
\text { et al. (2015); Lemos et al. } \\
\text { (2016) }\end{array}$ \\
\hline
\end{tabular}

\section{CELASTRACEAE} expectorante 
Monteverdia distichophylla bom-nome, bonone

Uterine inflammation, inflammations in general, healing $\mathrm{Sb}$

(Mart. ex Reissek) Biral bacupari

Gastritis

$\mathrm{Sb}$

Decoction

Yazbek et al. (2019)

Garcinia gardne
\& Triana) Zappi

\section{DILLENIACEAE}

Curatella americana L. Sambaiba, lixeira $\quad \operatorname{Tr} \quad \begin{aligned} & \text { general, anemia, depurative, tonic, bronchitis, } \\ & \text { indigestion, inflammation of the liver, muscle }\end{aligned}$

Curatella americana $\mathrm{L}$ sambaiba, lixeira $\quad \operatorname{Tr} \quad \begin{aligned} & \text { indigestion, inflammation of the liver, muscle } \\ & \text { ind }\end{aligned}$

Diarrhea, vaginal discharge, infections in general, anemia, depurative, tonic, bronchitis, cramp, intestinal colic, menstrual irregular,

$\mathrm{Sb}, \quad$ Decoction,

St, Fl, infusion, kidney pain, rehydration Ro natura

\begin{tabular}{|c|c|c|c|c|c|c|}
\hline & & & kidney pain, rehydration & & & \\
\hline $\begin{array}{l}\text { Doliocarpus dentatus (Aubl.) } \\
\text { Standl. }\end{array}$ & $\begin{array}{c}\text { cipó-de-cururu, cipó- } \\
\text { d'agua }\end{array}$ & $\mathrm{Cp}$ & Diuretic & $\mathrm{Sa}$ & in natura & Ribeiro et al. $\left(2017^{b}\right)$ \\
\hline
\end{tabular}

Erythroxylum vacciniifolium

catuaba, catuaba pau

Aphrodisiac, sexual impotence

Le, Infusion,

Ribeiro et al. (2014)

Mart.

\section{EUPHORBIACEAE}

Croton blanchetianus Baill.

marmeleiro, marmeleiro-branco

Sh

velame, velamebranco

Sh

Ectoparasites, dysentery, stomach pain, náusea

Nausea, indigestion, depurative, furuncle,

Croton heliotropiifolius Kunth

pião-brabo

Baill.

\section{FABACEAE}

Anadenanthera colubrina var. cebil (Griseb.) Altschul

\section{Bauhinia forficata Link.} ungulata
Bauhinia ungulata L. var.

Bowdichia virgilioides Kunth

\section{angico-preto, angico, angico-de-caroço, angico-branco}

pata-de-vaca, mororó pata-de-boi

sicupira, sucupira, sucupira-preta miroró, pata-de-vaca,

$\mathrm{Sb}$

maceration

Silva et al. (2014a); Souza et

$$
\text { al. (2014) }
$$

back pain, inflammations in general, itching,

Le,

Poultice

Souza et al. (2014); Macedo

influenza, cancer, fever, diarrhea, healing

Infusion,

et al. (2015)

$\mathrm{Sb}$ decoction

Silva et al. (2014a)

$\mathrm{Sb} \quad$ Maceration

Cough, bronchitis, anemia, inflammations in general, asthma, influenza, magical-religious use, whooping cough, lung inflammation, constipation, cancer, intestinal infections, depurative, blows, injury, scrofula,

diphtheria, foot cracks, gastritis, expectorant, stomach pain, swelling

Diabetes, depurative, rheumatism, pains in general, uterine inflammation, back pain, rheumatism, diuretic, kidney pain

Diarrhea, cancer, hypercholesterolemia, diabetes, obesity, constipation

$\mathrm{Sb}$,
$\mathrm{St}, \mathrm{Fl}$ Maceration, in St, Fl, 'cachaça'*, sirup
Le, Fr

Le, Fr

Fl, Decoction,

Le, infusion, in

$\mathrm{Sb} \quad$ 'cachaça'*

Decoction,

Le, Fl infusion,

maceration

Arthritis, osteoporosis, back pain, diabetes, rheumatism, indigestion, inflammations in general, uterine inflammation, healing, pains

$\mathrm{Sb}, \quad$ Maceration

Agra et al. (2007);

Albuquerque et a.1. (2007a),

Albuquerque et a.l. (2007b);

Pereira-Junior et al. (2014);

Ribeiro et al. (2014)

Silva et al. (2005); Bieski et al. (2015); Bolson et al. (2015); Yazbek et al. (2019)

Ribeiro et al. (2017b)

Albuquerque et a. (2007b); Souza et al. (2014); Ribeiro et al. (2017b) 
Copaifera langsdorffii Desf. paudoia, podoia, paud'óleo, copaiba

Dimorphandra gardneriana Tul. faveira, fava-d'anta

Dioclea grandiflora Mart. ex

Benth.

mucunã

jatobá-de-veado,

Hymenaea courbaril $\mathrm{L}$ jatobá, jatobá-de-boi jatobá-do-mato

Hymenaea stigonocarpa Mart. ex Hayne

jatobá-de-veado jatobá-do-cerrado in general, back pain, depurative, vaginal inflammation, throat inflammation, throat infection

Bronchitis, rheumatism, arthrosis, arthritis, leg pain, cough, healing, diarrhea, fever, allergy, swelling, influenza, headache, uterine inflammation, bone fracture, gastritis, angina,

blows, prostate inflammation, intestinal

inflammation, diuretic, uterine inflammation,

ovary infection, urinary infection, kidney

stones, kidney infection, snakebite, burns,

infections in general, throat inflammation,

repellent, constipation, cancer, depression,

stomach pain, gastritis, lung inflammation, anxiety

Inflammations in general, eye irritation,

cancer, pains in general, conjunctivitis,

cough, influenza, healing, hernia, marginal osteophytes

Prostate inflammation, healing, dermatitis

Gastritis, ulcer, cough, influenza, urethral problems, blows, anemia, asthma,

expectorant, inflammations in general, prostate inflammation, healing, constipation, nasal congestion, poisoning, blood problems, fever, headache, bronchitis, pains in general

Diarrhea, infections in general, prostate

cancer, anemia, depurative, leukemia,

anxiety, sedative, cataract, eye irritation, asthma, bronchitis, expectorant, pulmonary

tonic, influenza, pneumonia, gastritis,

indigestion, ulcer, tonic, inflammations in general, rheumatism, uterine inflammation

and ovary, vaginal cleaning, prostate

inflammation, kidney pain, healing, bone

fracture, pains in general, throat infection,

throat inflammation, vomit, tonic
Infusion, in

'cachaça'*,

decoction,

maceration, in

natura, sirup

$\mathrm{Se}$

$\mathrm{Fr}, \mathrm{Sb}$

Maceration,

decoction

Decoction,

infusion,

maceration

Decoction,

infusion, sirup,

maceration

$\mathrm{Fr}$

$\mathrm{Sb}$,

Fr,

Ro,

$\mathrm{Re}$,

$\mathrm{Sa}$

Decoction, maceration, infusion, poultice juice, sirup
Ribeiro et al. (2014); Souza et al. (2014); Macedo et al.

(2015); Saraiva et al. (2015);

Ribeiro et al. (2017b);

Guimarões et al. (2019)

Ribeiro et al. (2014); Souza et al. (2014); Macedo et al.

(2015)

Agra et al. (2007); Ribeiro et al. (2014)

Pereira-Junior et al. (2014); Ribeiro et al. (2014); Souza et al. (2014); Macedo et al.

(2015); Saraiva et al. (2015);

Ribeiro et al. (2017b)
Souza \& Felfili (2006) Ribeiro et al. (2017b); Migués et al. (2019) 
Libidibia ferrea (Mart. ex Tul.) L.P.Queiroz

pau-ferro, jucá

Machaerium acutifolium Vogel var. acutifolium

coração-de-nego

Mimosa tenuiflora (Willd.) Poir.

Mimosa verrucosa Benth.

Plathymenia reticulata Benth.

jurema-preta amargoso, amarelo, candeia, vinhático

Stryphnodendron rotundifolium

Mart.

barbatimão, barbatenã
Anemia, asthma, vaginal cleaning, blows, stroke, diarrhea, stomach pain, injury, internal wounds, insect bites, throat inflammation,

$\operatorname{Tr}$ (n)

cracks, cough, ulcer, back pain, worms,

inflammation of internal and external organs, bone pain, bone fracture

$\operatorname{Tr}$

Pains in general, inflammation of internal and external organs

Bronchitis, cough, aphrodisiac, uterine

inflammation, wounds, pains in general,

inflammation of external organs Uterine inflammation

Bleeding, swelling, pains in the liver, kidney pain, healing

Diabetes, ulcer, healing, inflammations in general, genital disease, bleeding, worms,

hypertension, anemia, cancer, hepatic problems, gastritis, uterine inflammation and

ovary, respiratory distress, furuncle, urinary

infection, dermatitis, throat infection

Vachellia farnesiana (L.) Wigh

\section{\& Arn.}

Vismia guianensis (Aubl.)

Choisy

LAMIACEAE

Rhaphiodon echinus Schauer

acácia, coronha

$\mathrm{Sh}$

Anemia, hypercholesterolemia, diabetes, chest pain, premature ejaculation

.

Back pain, pains in general, kidney pain magical-religious use

lacre, lacre-vermelho

$\operatorname{Tr}$

Influenza, intestinal colic

in diseases, infections in general,

ysentery, genital disease, mycoses, furuncle,

vaginal discharge, sífilis, worms, cancer,

depurative, diabetes, obesity, abscess,

hemorrhoids, edema, labyrinthitis,

LYTHRACEAE

romã-braba,

Lafoensia pacari A.St.-Hil. lagartixeiro, pacari, didal
$\mathrm{Sh}$ pneumonia, tuberculosis, heartburn, pains in
the liver, gastritis, indigestion, gallbladder stones, ulcer, back pain, uterine

inflammation, diuretic, uterine and ovarian

infection, kidney infection, menopause
In 'cachaça'*,

maceration,

banho, infusion,

decoction, sirup,

in natura

$\mathrm{Sb}, \quad$ Decoction, in

Ro 'cachaça'*, seco

$\mathrm{Sb}$ Decoction, sirup infusion

\section{Decoction}

Decoction,

maceration

Maceration, in

'cachaça'*,

infusion,

decoction, sirup
Ribeiro et al. (2014); Silva et al. (2014a); Magno-Silva et al. (2020)

\section{Ribeiro et al. (2014)}

Albuquerque (2001); Agra et al. (2007); Ribeiro et al.

(2014); Macedo et al. (2015) Aguiar \& Barros (2012)

Ribeiro et al. (2017b)

Ribeiro et al. (2014); Souza et al. (2014); Bitu et al. (2015); Macedo et al. (2015)

Fl, Maceration infusion

Ribeiro et al. (2017b)

Le

infusion

Le Uninformed Albuquerque et al. (2017b)

disorders, healing, burns, headache

\section{MALPIGHIACEAE}


Journal of Environmental Analysis and Progress V. 06 N. 04 (2021) 326-351

\begin{tabular}{|c|c|c|c|c|c|c|}
\hline Byrsonima sericea DC. & $\begin{array}{l}\text { murici-vermelho, } \\
\text { murici-branco }\end{array}$ & $\operatorname{Tr}$ & Hypercholesterolemia, healing & $\mathrm{Sb}$ & Maceration & Ribeiro et al. (2014) \\
\hline \multicolumn{7}{|l|}{ MALVACEAE } \\
\hline Guazuma ulmifolia Lam. & $\begin{array}{l}\text { cabeça-de-negro, } \\
\text { chico-magro, } \\
\text { mutamba }\end{array}$ & $\operatorname{Tr}$ & $\begin{array}{c}\text { Snakebite, diabetes, gastritis, hair tonic, } \\
\text { wounds }\end{array}$ & $\mathrm{Sb}$ & $\begin{array}{l}\text { In 'cachaça'*, } \\
\text { decoction, } \\
\text { maceration }\end{array}$ & $\begin{array}{l}\text { Ribeiro et al. (2014); Ribeiro } \\
\text { et al. (2017b) }\end{array}$ \\
\hline $\begin{array}{l}\text { Pavonia malacophylla (Link \& } \\
\text { Otto) Garcke }\end{array}$ & malva-branca & $\mathrm{S} 1$ & Cough, influenza & $\begin{array}{l}\text { Le, } \\
\text { Ro }\end{array}$ & sirup & Ribeiro et al. (2014) \\
\hline Sida cordifolia $\mathrm{L}$. & $\begin{array}{l}\text { malva-branca, malva- } \\
\text { veludo, malvão }\end{array}$ & Sl & $\begin{array}{c}\text { Acne, vaginal discharge, cough, toothache, } \\
\text { inflammations in general, itching, wounds, } \\
\text { indigestion, magical-religious use, influenza, } \\
\text { mycoses, irregular menstruation }\end{array}$ & $\mathrm{Le}$ & Infusion, sirup & $\begin{array}{l}\text { Agra et al. (2007); } \\
\text { Albuquerque et al. (2007b); } \\
\text { Santana et al. (2016) }\end{array}$ \\
\hline Sida spinosa $\mathrm{L}$. & $\begin{array}{l}\text { malva-lanceta, malva- } \\
\text { relógio }\end{array}$ & $\mathrm{He}$ & $\begin{array}{l}\text { Asthma, influenza, respiratory problems in } \\
\text { general, ulcers, insect bites, emollient }\end{array}$ & $\mathrm{Le}$ & Infusion & Agra et al. (2007) \\
\hline $\begin{array}{l}\text { Sidastrum micranthum (A.St.- } \\
\text { Hil.) Fryxell }\end{array}$ & guaxima, malva-preta & Sh & Bronchitis, cough, asthma, emollient & $\begin{array}{l}\text { Le, } \\
\mathrm{Wp}\end{array}$ & Infusion, poultice & Agra et al. (2007) \\
\hline \multicolumn{7}{|l|}{ MELASTOMATACEAE } \\
\hline Miconia albicans (Sw.) Triana & $\begin{array}{l}\text { candieiro, carrasco, } \\
\text { canela-de-veio }\end{array}$ & Sh & $\begin{array}{l}\text { Fever, vitiligo, magical-religious use, } \\
\text { arthritis, back pain }\end{array}$ & St, Le & $\begin{array}{l}\text { Infusion, } \\
\text { decoction, } \\
\text { maceration }\end{array}$ & $\begin{array}{l}\text { Albuquerque et al. (2007b), } \\
\text { Ribeiro et al. (2017b) }\end{array}$ \\
\hline \multicolumn{7}{|l|}{ MYRTACEAE } \\
\hline Myrcia splendens (Sw.) DC. & $\begin{array}{l}\text { murta, sangue-de- } \\
\text { tatu, cumatí }\end{array}$ & $\operatorname{Tr}$ & Cancer & $\begin{array}{l}\mathrm{Sb} \\
\mathrm{Sa}\end{array}$ & $\begin{array}{l}\text { In natura, } \\
\text { maceration }\end{array}$ & Ribeiro et al. (2017b) \\
\hline Psidum myrsinites DC. & $\begin{array}{l}\text { araçá-vermelho, } \\
\text { goiabinha }\end{array}$ & $\operatorname{Tr}$ & Stomach pain, diarrhea & $\begin{array}{l}\mathrm{Le}, \\
\mathrm{Sb}, \mathrm{Fr}\end{array}$ & $\begin{array}{l}\text { Infusion, } \\
\text { Maceration }\end{array}$ & Ribeiro et al. (2014) \\
\hline $\begin{array}{l}\text { NYCTAGINACEAE } \\
\text { Guapira graciliflora (Mart. ex } \\
\text { Schmidt) Lundell }\end{array}$ & pau-piranha & $\operatorname{Tr}$ & $\begin{array}{c}\text { Placental delivery, mastite, wounds, } \\
\text { infections in general }\end{array}$ & $\begin{array}{l}\mathrm{Sb}, \\
\mathrm{Le}\end{array}$ & $\begin{array}{l}\text { Infusion, } \\
\text { decoction }\end{array}$ & Silva et al. (2020) \\
\hline \multicolumn{7}{|l|}{ OLACACEAE } \\
\hline Ximenia americana $\mathrm{L}$. & ameixa & Sh & $\begin{array}{l}\text { Obesity, healing, diabetes, cough, hoarseness, } \\
\text { constipation, genital disease, osteoporosis, } \\
\text { gastritis, uterine inflammation, burning, } \\
\text { itching, furuncle, throat inflammation, pains } \\
\text { in the liver, dermatitis, back pain, genital } \\
\text { inflammation, inflammation of internal } \\
\text { organs, kidney pain, contusion, gallbladder } \\
\text { problems, prostate inflammation, blows, } \\
\text { influenza, headache, fever }\end{array}$ & $\begin{array}{l}\mathrm{Sb} \\
\text { Ro, } \\
\text { Fr, St }\end{array}$ & $\begin{array}{l}\text { Infusion, } \\
\text { maceration, } \\
\text { decoction, in } \\
\text { 'cachaça'* }\end{array}$ & $\begin{array}{l}\text { Ribeiro et al. (2014), Souza et } \\
\text { al. (2014); Bitu et al. (2015); } \\
\text { Macedo et al. (2015) }\end{array}$ \\
\hline
\end{tabular}




\begin{tabular}{|c|c|c|c|c|c|c|}
\hline $\begin{array}{l}\text { Passiflora cincinnata Mast. } \\
\text { Passiflora foetida } \mathrm{L} \text {. }\end{array}$ & $\begin{array}{l}\text { maracujá-do-mato, } \\
\text { maracujá-de-boi } \\
\text { maracujá-do-estalo }\end{array}$ & $\mathrm{Cp}$ & $\begin{array}{c}\text { Hypertension, anxiety, sedative, insomnia, } \\
\text { renal insufficiency } \\
\text { Gonorrhea }\end{array}$ & $\begin{array}{l}\text { Le, } \\
\text { Fr, } \\
\text { Ro } \\
\mathrm{Wp}\end{array}$ & $\begin{array}{l}\text { Decoction, juice, } \\
\text { infusion } \\
\text { decoction }\end{array}$ & $\begin{array}{c}\text { Ribeiro et al. (2014); Souza et } \\
\text { al. (2014); Macedo et al. } \\
\text { (2015) } \\
\text { Agra et al. (2007) }\end{array}$ \\
\hline \multicolumn{7}{|l|}{ PLANTAGINACEAE } \\
\hline Scoparia dulcis L. & $\begin{array}{l}\text { vassourinha, } \\
\text { vassourinha-de- } \\
\text { benzer }\end{array}$ & $\mathrm{He}$ & $\begin{array}{l}\text { Irregular menstruation, worms, magical- } \\
\text { religious use, bladder wound, healing, } \\
\text { diabetes, pains in general, bone fracture, } \\
\text { swelling in pregnant woman, kidney pain, } \\
\text { syphilis, fever, bronchitis, throat } \\
\text { inflammation, cough, influenza, toothache, } \\
\text { renal inflammation, infections in general, } \\
\text { depurative, tonic for the heart, conjunctivitis, } \\
\text { pneumonia, gastritis, inflammations in } \\
\text { general, muscle cramp, uterine inflammation, } \\
\text { vaginal discharge }\end{array}$ & $\begin{array}{l}\text { Wp, } \\
\text { Ro, } \\
\text { Le }\end{array}$ & $\begin{array}{c}\text { Decoction, } \\
\text { infusion, } \\
\text { decoction, } \\
\text { maceration, sirup, } \\
\text { juice }\end{array}$ & $\begin{array}{c}\text { Agra et al. (2007); } \\
\text { Albuquerque et al. (2007b); } \\
\text { Bieski et al. (2012); Ribeiro et } \\
\text { al. (2014); Silva et al. } \\
\text { (2014a); Souza et al. (2014); } \\
\text { Lemos et al. (2016); Ribeiro } \\
\text { et al. (2017b) }\end{array}$ \\
\hline \multicolumn{7}{|l|}{ PLUMBAGINACEAE } \\
\hline Plumbago scandens $\mathrm{L}$. & louco & $\mathrm{He}$ & Pains in general, arthritis, sedative, warts & $\mathrm{Wp}$ & $\begin{array}{c}\text { Decoction, } \\
\text { infusion, poultice }\end{array}$ & Agra et al. (2007) \\
\hline \multicolumn{7}{|l|}{ POLYGALACEAE } \\
\hline $\begin{array}{l}\text { Bredemeyera brevifolia (Benth.) } \\
\text { Klotzsch ex A.W.Benn. }\end{array}$ & $\begin{array}{l}\text { manacá-cipó, mau- } \\
\text { vizinho }\end{array}$ & $\mathrm{Cp}$ & Kidney pain, back pain & $\mathrm{Sb}$ & Maceration & Ribeiro et al. (2014) \\
\hline Bredemeyera floribunda Willd. & pau-gemada & $\operatorname{Tr}$ & Tonic, stomach problems & $\begin{array}{l}\mathrm{Sb}, \\
\mathrm{Ro}\end{array}$ & Maceration & Ribeiro et al. (2014) \\
\hline
\end{tabular}

PROTEACEAE
Anxiety, sedative, menstrual colic,

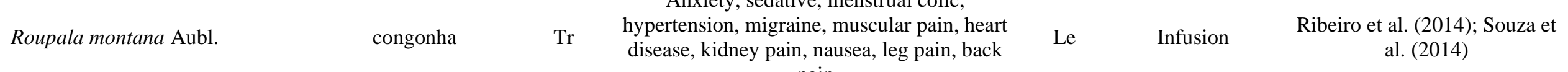

\section{RHAMNACEAE} pain

\begin{tabular}{|c|c|c|c|c|c|c|}
\hline \multicolumn{7}{|l|}{ RHAMNACEAE } \\
\hline Colubrina cordifolia Reissek & $\begin{array}{l}\text { saboeiro, joão- } \\
\text { vermelho }\end{array}$ & Sh & Healing & Le & Maceration & Ribeiro et al. (2014) \\
\hline \multicolumn{7}{|l|}{ RUBIACEAE } \\
\hline Borreria verticillata (L.) G.Mey & vassourinha-de-botão & $\mathrm{He}$ & $\begin{array}{l}\text { Hemorrhoids, vaginal discharge, worms, } \\
\text { sexual impotence, magical-religious use }\end{array}$ & $\begin{array}{l}\text { Wp, } \\
\text { Le, } \\
\text { Ro }\end{array}$ & Decoction & $\begin{array}{l}\text { Agra et al. (2007); } \\
\text { Albuquerque et al. (2007b) }\end{array}$ \\
\hline Chiococca alba (L.) Hitchc. & caninana, cainca & Sh & $\begin{array}{l}\text { Headache, snakebite, infections in general, } \\
\text { leprosy, intestinal infections, syphilis, } \\
\text { influenza, gastritis, constipation, back pain, } \\
\text { inflammations in general, rheumatism, }\end{array}$ & $\begin{array}{l}\text { Le, } \\
\text { Ro }\end{array}$ & $\begin{array}{l}\text { Infusion, } \\
\text { decoction, } \\
\text { maceration }\end{array}$ & $\begin{array}{l}\text { Ribeiro et al. (2017b); } \\
\text { Miguéis et al. (2019) }\end{array}$ \\
\hline
\end{tabular}




\begin{tabular}{|c|c|c|c|c|c|c|}
\hline & & & $\begin{array}{l}\text { vaginal discharge, sexual impotence, prostate } \\
\text { inflammation, healing, pains in general, fever }\end{array}$ & & & \\
\hline Cordiera sessilis (Vell.) Kuntze & marmelinho & Sh & Diarrhea & $\begin{array}{l}\mathrm{Sb} \\
\mathrm{Ro}\end{array}$ & Maceration & Ribeiro et al. (2017b) \\
\hline $\begin{array}{l}\text { Guettarda viburnoides Cham. \& } \\
\text { Schltdl. }\end{array}$ & angélica & Sh & Pains in general, throat inflammation & $\begin{array}{l}\mathrm{Sb} \\
\mathrm{Le}\end{array}$ & $\begin{array}{l}\text { Infusion, } \\
\text { decoction }\end{array}$ & Ribeiro et al. (2014) \\
\hline $\begin{array}{l}\text { Tocoyena formosa (Cham. \& } \\
\text { Schltdl.) K.Schum. }\end{array}$ & $\begin{array}{l}\text { genipapinho, } \\
\text { geninapo-bravo }\end{array}$ & Sh & $\begin{array}{l}\text { Rheumatism, bone fracture, contusion, blows, } \\
\text { swelling, healing }\end{array}$ & $\begin{array}{l}\text { Le, } \\
\mathrm{Sb}, \\
\mathrm{Re}\end{array}$ & $\begin{array}{l}\text { Poultice, } \\
\text { maceration }\end{array}$ & $\begin{array}{l}\text { Agra et al. (2007); Ribeiro et } \\
\text { al. (2014); Souza et al. (2014) }\end{array}$ \\
\hline \multicolumn{7}{|l|}{ RUTACEAE } \\
\hline Zanthoxylum gardneri Engl. & $\begin{array}{l}\text { laranjinha, } \\
\text { limãozinho, quebra- } \\
\text { faca }\end{array}$ & $\operatorname{Tr}$ & Injury, influenza, diarrhea, headache & $\mathrm{Sb}$ & $\begin{array}{l}\text { Infusion, } \\
\text { maceration }\end{array}$ & Ribeiro et al. (2014) \\
\hline Zanthoxylum rhoifolium Lam. & $\begin{array}{l}\text { laranja-braba, } \\
\text { mamica-de-porca }\end{array}$ & $\operatorname{Tr}$ & $\begin{array}{l}\text { Depurative, ulcer, gastritis, healing, throat } \\
\text { inflammation }\end{array}$ & $\mathrm{Sb}, \mathrm{Fr}$ & $\begin{array}{l}\text { Decoction, } \\
\text { maceration }\end{array}$ & $\begin{array}{c}\text { Ribeiro et al. (2017b); Yazbek } \\
\text { et al. (2019) }\end{array}$ \\
\hline \multicolumn{7}{|l|}{ SALICACEAE } \\
\hline Casearia sylvestris $\mathrm{Sw}$. & $\begin{array}{l}\text { pereirinha, chá-de- } \\
\text { frade }\end{array}$ & $\operatorname{Tr}$ & Diabetes & $\mathrm{Sb}$ & $\begin{array}{l}\text { Decoction, } \\
\text { infusion, } \\
\text { maceration, sirup, } \\
\text { in natura }\end{array}$ & Ribeiro et al. (2017b) \\
\hline \multicolumn{7}{|l|}{ SAPINDACEAE } \\
\hline Magonia pubescens A.St.- Hil. & tingui & $\operatorname{Tr}$ & Stingray sting, dermatitis, seborreia & $\mathrm{Sb}$ & Poultice & $\begin{array}{l}\text { Souza \& Felfiti (2006); } \\
\text { Ribeiro et al. (2017b) }\end{array}$ \\
\hline Matayba guianensis Aubl. & pitomba-braba & $\operatorname{Tr}$ & Leg pain, back pain & $\mathrm{Sb}$ & Uninformed & Souza et al. (2014) \\
\hline Serjania lethalis A.St.-Hil. & $\begin{array}{l}\text { chiador, cipó-de- } \\
\text { vaqueiro, cruapé }\end{array}$ & $\mathrm{Cp}$ & Toothache & $\mathrm{Sb}$ & Uninformed & Souza et al. (2014) \\
\hline $\begin{array}{l}\text { Talisia esculenta (Cambess.) } \\
\text { Radlk. }\end{array}$ & pitomba & $\operatorname{Tr}$ & Kidney stones & $\mathrm{Fr}$ & Maceration & Ribeiro et al. (2017b) \\
\hline \multicolumn{7}{|l|}{ SIMAROUBACEAE } \\
\hline Simarouba versicolor A.St.- Hil. & mata-menino & $\operatorname{Tr}$ & Itching, healing & $\begin{array}{l}\mathrm{Sb} \\
\mathrm{Le}\end{array}$ & $\begin{array}{l}\text { Decoction, } \\
\text { maceration }\end{array}$ & Ribeiro et al. (2017b) \\
\hline \multicolumn{7}{|l|}{ SIPARUNACEAE } \\
\hline Siparuna guianensis Aubl. & $\begin{array}{l}\text { pau-fedido, capitiú, } \\
\text { negramina }\end{array}$ & $\operatorname{Tr}$ & $\begin{array}{l}\text { Influenza, sinusitis, respiratory problems in } \\
\text { general, stroke, ulcer, itching, arthritis, } \\
\text { rheumatism, pains in general, fever, repellent, } \\
\text { magical-religious use }\end{array}$ & Le & $\begin{array}{l}\text { Decoction, } \\
\text { infusion, } \\
\text { maceration }\end{array}$ & $\begin{array}{l}\text { Pagani et al. (2017); Ribeiro } \\
\text { et al. (2017b) }\end{array}$ \\
\hline \multicolumn{7}{|l|}{ SMILACACEAE } \\
\hline Smilax campestris Griseb. & japecanga & $\mathrm{Cp}$ & $\begin{array}{l}\text { Dermatitis, syphilis, rheumatism, depurative, } \\
\text { diuretic, arthritis, diaphoretic }\end{array}$ & Ro & $\begin{array}{l}\text { Decoction, } \\
\text { infusion }\end{array}$ & Rodrigues \& Carvalho (2001) \\
\hline $\begin{array}{l}\text { SOLANACEAE } \\
\text { Solanum rhytidoandrum Sendtn. }\end{array}$ & jurebeba-branca & Sh & Hepatic problems & Ro & Decoction & Agra et al. (2007) \\
\hline
\end{tabular}

Cruz, R.P.; Almeida-Bezerra, J.W.; Menezes, S.A.; Silva, V.B.; Santos, L.T.; Morais-Braga, M.F.B.; Moraes, J.L. 338 
Journal of Environmental Analysis and Progress V. 06 N. 04 (2021) 326-351

\section{TURNERACEAE}

Turnera subulata $\mathrm{Sw}$.

perpétua, chanana

$\mathrm{He}$

Irregular menstruation, expectorant, bronchitis, cough

Ro, Decoction, nfusion, sirup

Agra et al. (2007); Lemos et

$\mathrm{Wp}$

\section{VERBENACEAE}

Lantana camara L.

camará, chumbinho

alecrim-pimenta, alecrim-de-flepa,

Lippia microphylla Cham.

\section{VITACEAE} salva-de-marajó
$\mathrm{Sh}$

Diuretic, expectorant, cough, irregular menstruation, pains in general, rheumatism

Sh

Antiseptic, respiratory problems in general, myiasis, stomach pain, gastritis, malaria

$\begin{array}{cl}\text { Le, Fl Decoction } & \\ \text { Le } & \begin{array}{c}\text { Decoction, } \\ \text { infusion, }\end{array}\end{array}$

Agra et al. (2007); Ribeiro et al. (2014); Souza et al. (2014)

Agra et al. (2007); Silva et al. (2014a); Vásquez et al. (2014)

\section{Cissus simsiana Schult. \&}

Schult.f.

parreira

myiasis

$\mathrm{Sb}$

In natura

Silva et al. (2014a)

\section{VOCHYSIACEAE}

Qualea parviflora Mart.

\section{$\mathrm{Sb}$ Decoction,}

Diarrhea, anemia, diabetes, conjunctivitis,

ulcer, inflammations in general, kidney infection, internal wounds, throat infection

Le, infusion,

Ro maceration


When distributing the therapeutic indications attributed to the most versatile species according to the ICD-10 body systems of the World Health Organization (WHO), we observed that diseases of the digestive system (DDS), musculoskeletal system, and connective tissue (DMC), genitourinary system (DGS) and skin diseases (DS) are the disorders most cited and with the largest number of species used for treatment (10 species), as shown in Table 2. Similar data were found in the study of Ferreira-Júnior et al. (2015), where diseases of the digestive system and genitourinary system were also prevalent in research conducted in communities in Chapada of Araripe, reinforcing the importance of many native species in the treatment of diseases grouped in these body systems.
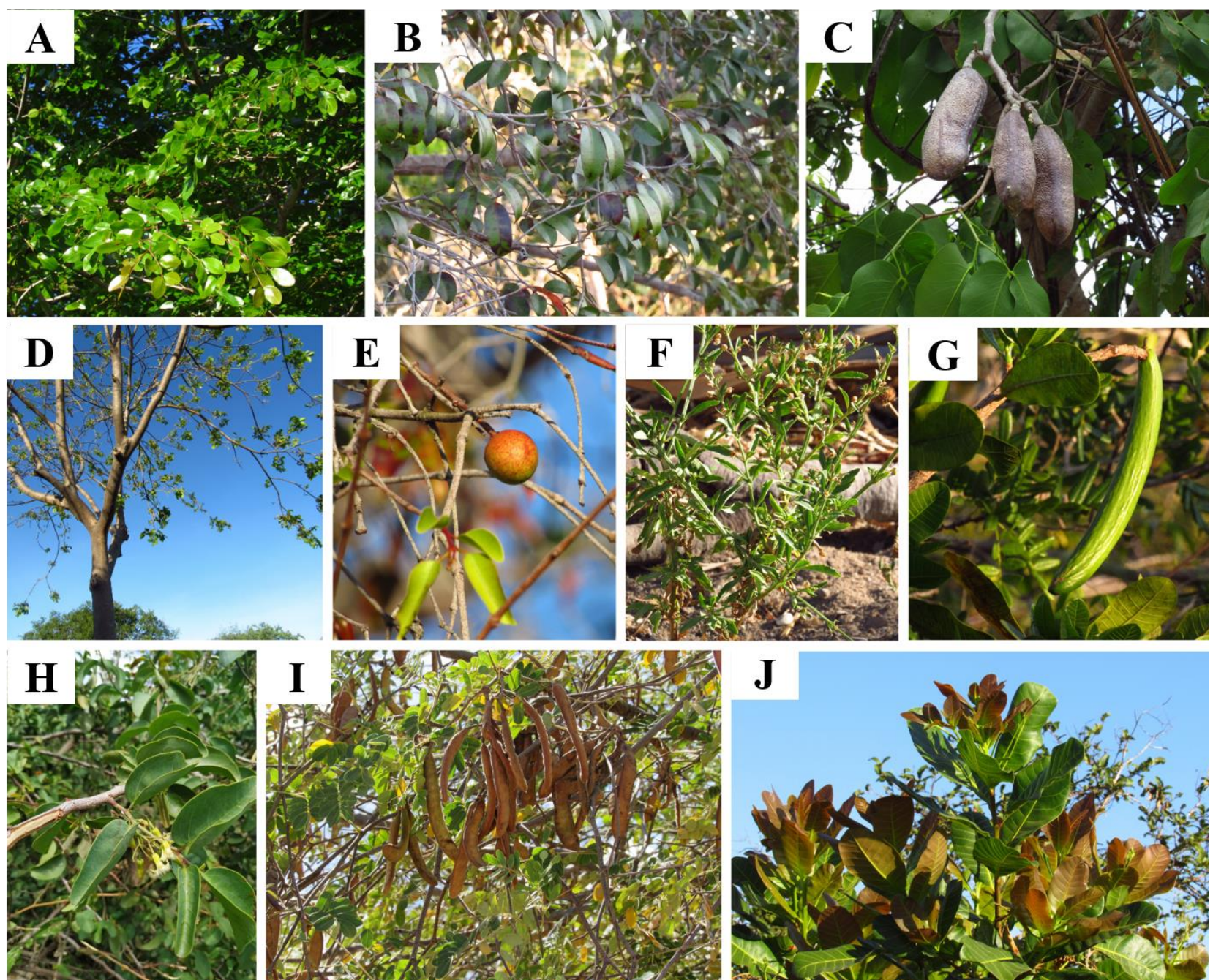

Figure 5. Species of medicinal use of Chapada of Araripe (Brazil), with greater numbers of therapeutic indications. $\mathrm{A}=$ Copaifera langsdorffii (pau-d'óleo); $\mathrm{B}=$ Lafoensia pacari (romã braba); $\mathrm{C}=$ Hymenaea stigonocarpa (jatobá-do-cerrado); $\mathrm{D}=$ Astronium urundeuva (aroeira); $\mathrm{E}=$ Hancornia speciosa (mangaba); $\mathrm{F}$ = Scoparia dulcis (vassourinha); $\mathrm{G}=$ Himatanthus drasticus (janaguba); $\mathrm{H}=$ Ximenia americana (ameixa); $\mathrm{I}$ = Libidibia ferrea (pau-ferro); J = Anacardium occidentale (caju). Font: Cruz, R. P. (2021). 
Table 2. List of versatile species found in Chapada of Araripe (Brazil) with their respective chemical groups and pharmacological and biological activities. Body systems =Diseases of the digestive system (DDS); Diseases of the respiratory system (DRS); Diseases of the genitourinary system (DGS); Injury, poisoning, and certain other infirmities with external causes (IPO); Diseases of the musculoskeletal system and connective tissues (DMC); Endocrine, nutritional and metabolic diseases (ENM); Diseases of the skin and subcutaneous tissues (DSS); Diseases of the circulatory system (DCS); Diseases of the nervous system (DNS); Infectious and parasitic diseases (IPD); Diseases of the ears and mastoid processes (DEM); Neoplasms (NEO); Diseases of the eyes and adnexa (DEA); Mental and behavioral disorders (MBD); Afflictions and pains not defined (AND). RI = Relative Importance. Font: Cruz et al. (2021).

\begin{tabular}{|c|c|c|c|c|c|}
\hline Species & Body systems & $\mathbf{R I}$ & Chemical groups & $\begin{array}{c}\text { Pharmacological and } \\
\text { biological activities }\end{array}$ & References \\
\hline $\begin{array}{c}\text { Anacardium } \\
\text { occidentale } \mathrm{L} .\end{array}$ & $\begin{array}{l}\text { DGS, DCS, } \\
\text { NC, DSS, SD, } \\
\text { DMC, ENM, } \\
\text { IPD, IPO }\end{array}$ & 1.38 & $\begin{array}{l}\text { Alkaloids, flavonoids, saponins, tannins, } \\
\text { anthraquinones, terpenoids, cardiac glycosides, } \\
\text { phenolic compounds, steroids, carotenoids, } \\
\text { coumarins, anthocyanidins, monoterpenes, } \\
\text { sesquiterpenes }\end{array}$ & $\begin{array}{l}\text { Antioxidant, lipid-lowering, } \\
\text { antibacterial, anti- } \\
\text { inflammatory, healing, } \\
\text { antiparasitic, anti-tumor }\end{array}$ & $\begin{array}{l}\text { Aguilar et al. (2012); Mustapha } \\
\text { et al. (2015); Vasconcelos et al. } \\
\text { (2015); Anyaegbu et al. (2017); } \\
\text { Davuluri et al. (2019); } \\
\text { Aponjolosun \& Fasola (2020); } \\
\text { Costa et al. (2020) }\end{array}$ \\
\hline $\begin{array}{l}\text { Astronium } \\
\text { urundeuva (M. } \\
\text { Allemão) Engl. }\end{array}$ & $\begin{array}{l}\text { AND, DRS, } \\
\text { DSS, DGS, } \\
\text { DDS, DMC, } \\
\text { NEO, DR, IPD, } \\
\text { ENM, IPO, } \\
\text { DCS }\end{array}$ & 1.86 & $\begin{array}{l}\text { Polyphenols, flavonoids, chalcones, fatty acids, } \\
\text { monoterpenes, sesquiterpenes, tannins, } \\
\text { anthocyanins, anthocyanidins, flavones, } \\
\text { flavonols, xanthones, auronas, flavononols, } \\
\text { leucoanthocyanidins, catechins, flavonones }\end{array}$ & $\begin{array}{l}\text { Anti-inflammatory, } \\
\text { gastroprotective, antibacterial, } \\
\text { anti-tumor, anti-parasitic }\end{array}$ & $\begin{array}{l}\text { Figueredo et al. (2014); Araújo } \\
\text { et al. (2017); Carvalho et al. } \\
\text { (2017); Galvão et al. (2018); } \\
\text { Castro et al. (2020) }\end{array}$ \\
\hline $\begin{array}{l}\text { Copaifera } \\
\text { langsdorffii Desf. }\end{array}$ & $\begin{array}{l}\text { IPO, DNS, } \\
\text { DGS, DCS, } \\
\text { IPD, NEO, } \\
\text { MBD, DRS, } \\
\text { DMC, DSS, } \\
\text { DDS, AND } \\
\text { IPO, DGS, }\end{array}$ & 2.00 & Flavonoids, sesquiterpenes, diterpenes & $\begin{array}{l}\text { Gastroprotective, anti- } \\
\text { inflammatory, antioxidant, } \\
\text { antipsoriatic, healing }\end{array}$ & $\begin{array}{l}\text { Pereira et al. (2008); Gelmini et } \\
\text { al. (2013); Lemos et al. (2015); } \\
\quad \text { Gushiken et al. (2017) }\end{array}$ \\
\hline $\begin{array}{l}\text { Hancornia } \\
\text { speciosa } \text { Gomes }\end{array}$ & $\begin{array}{l}\text { DDS, DCS, } \\
\text { AND, NEO, } \\
\text { DSS, IPD, } \\
\text { ENM, DEM, } \\
\text { DEA, DMC }\end{array}$ & 1.81 & $\begin{array}{c}\text { Phenols, tannins, flavones, flavonols, xanthones, } \\
\text { leucoanthocyanidins, catechins, flavonones, } \\
\text { alkaloids }\end{array}$ & $\begin{array}{l}\text { Antioxidant, antimutagen, } \\
\text { osteogenic, anti-diabetic, } \\
\text { antimicrobial, cytotoxic }\end{array}$ & $\begin{array}{l}\text { Assumpção et al. (2014); Lima } \\
\text { et al. (2015); Pereira et al. } \\
\text { (2015); Floriano et al. (2016); } \\
\text { Santos et al. (2016) }\end{array}$ \\
\hline $\begin{array}{l}\text { Himatanthus } \\
\text { drasticus (Mart.) } \\
\quad \text { Plumel }\end{array}$ & $\begin{array}{l}\text { DDS, NEO, } \\
\text { DSS, IPD, } \\
\text { AND, DMC, } \\
\text { DNS, DGS, } \\
\text { DRS, ENM, } \\
\text { DCS }\end{array}$ & 1.62 & $\begin{array}{l}\text { Triterpenes, steroids, saponins, monoterpenes, } \\
\text { sesquiterpenes, flavonoids, iridoides }\end{array}$ & $\begin{array}{l}\text { Cytotoxic, antinociceptive, } \\
\text { antitumor, anti-inflammatory, } \\
\text { genotoxic, antibacterial, anti- } \\
\text { diabetic }\end{array}$ & $\begin{array}{l}\text { Colares et al. (2008); Sousa et } \\
\text { al. (2010); Silva et al. (2017); } \\
\text { Almeida et al. (2019); Moura et } \\
\text { al. (2020); Morais et al. (2020) }\end{array}$ \\
\hline
\end{tabular}


DDS, IPD,

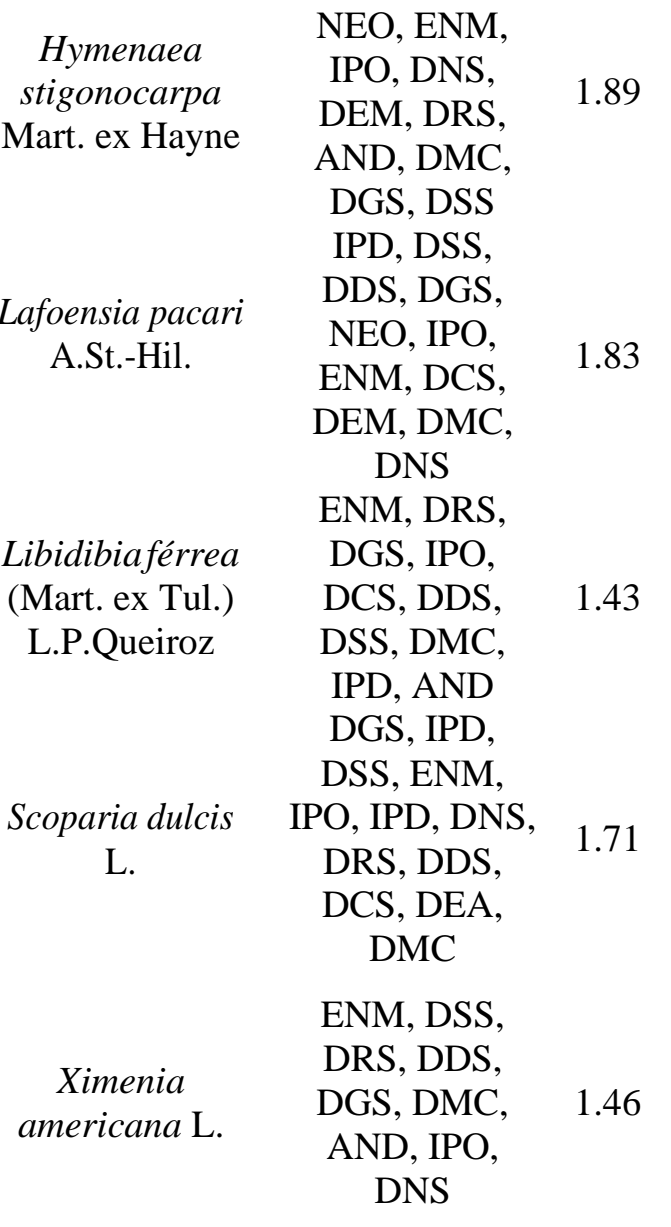

Flavonoids, tannins, terpenes, coumarins, fatty acids, flavones

Saponins, tannins, steroids, triterpenes, flavonoids

Tannins, flavonoids, alkaloids, cinnamic derivatives, triterpenes, saponins, organic acids, phenols, lactone sesquiterpenes, anthraquinones

Diterpenes, glycosides, phenolic compounds, steroids, triterpenes, flavonoids

Alkaloids, flavonoids, lignans, monoterpenes, sesquiterpenes, diterpenes, naphthoquinones, saponins, hydrolyzable tannins, triterpenes, steroids, polyphenols, anthocyanins, aurones, leucoanthocyanidin, catechins, anthocyanidins flavones, chalcones
Gastroprotective, healing, antioxidant, intestinal antiinflammatory, anti-thermal, antibacterial

Gastroprotective, antitumor, antidepressant, antimutagenic, antigenotoxic, cytotoxic, antiinflammatory, antioxidant, healing

Antibacterial, antiinflammatory, antioxidant, antinociceptive, antiparasitic, healing activity

Anti-inflammatory, antitumor, antioxidant, antidiabetic, antimicrobial

Muthumani et al. (2010); Mishra

Gastroprotective, antiparasitic, antioxidant, anti-

inflammatory, antinoceptive, antidiabetic activity et al. (2013); Parvataneni

(2019); Nur-e-Alam et al. (2020)

Orsi et al. (2012); Dimech et al. (2013); Maranhão et al. (2013);

Orsi et al. (2014); Silva et al. (2014b)

Tamashiro-Filho et al. (2012); Lima et al. (2013); Galdino et al.

(2015); Pereira et al. (2018);

Cordeiro et al. (2019); Chaibub et al. (2020)

Kobayashi et al. (2015);

Comandolli-Wyrepkowski et al.

(2017); Falcão et al. (2019);

Luna et al. (2020)

Almeida et al. (2016); Silva-

Leite et al. (2017); Shettar \&

Vedamurthy (2017); Sobeh et al.

(2017); Aragão et al. (2018);

Silva et al. (2018); Menezes et al. (2019) 
When relating the therapeutic indications of the species with their biological and pharmacological activities available in the literature, we verify the therapeutic potential of all versatile medicinal species found in this study. Species indicated for disorders of the digestive system, such as stomach pain, ulcer, diarrhea, and gastritis, have reports of gastroprotective activity: C. langsdorffii (Lemos et al., 2015), L. pacari (Tamashiro-Filho et al., 2012; Chaibub et al., 2020), H. stigonocarpa (Orsi et al., 2012), A. urundeuva (Galvão et al., 2018) and X. americana (Aragão et al., 2018). All these species, when submitted to preclinical tests (in vivo), were able to reduce the formation of gastric ulcers in animal models (Orsi et al., 2012; Tamashiro-Filho et al., 2012; Lemos et al., 2015; Aragão et al., 2018; Galvão et al., 2018; Chaibub et al., 2020), reinforcing the evidence of pharmacological properties of these species used in traditional medicine.

The inflammatory process was another condition with many citations in the group of unclassified systems or pain; we verified investigations of anti-inflammatory actions in the species A. occidentale (Vasconcelos et al., 2015) and A. urundeuva (Galvão et al., 2018) by significantly inhibiting ear edema in mice and rats respectively. C. langsdorffii (Pereira et al., 2008) and $H$. drasticus (Almeida et al., 2019) also due to inhibition of leg edema in animal models, and $L$. ferrea, which in addition to anti-inflammatory properties, also had an anti-receptive effect in vivo test (Falcão et al., 2019).

Anti-tumor activities have also been observed in plants associated with the popular treatment of neoplasms. A. urundeuva, in an in vitro test against HeLa, HEK-293, and Vero E6 cells, demonstrated mild anti-tumor activity in cancer cells and did not present toxicity in human cells (Araújo et al., 2007). H. drasticus also had an anticancer effect compared to the experimental model Sarcoma 180 (Souza et al., 2010). However, in the study of Moura et al. (2020), the latex of $H$. drasticus was genotoxic to S-180 cells in low concentrations $\left(\geq 50 \mu \mathrm{g} \mathrm{mL}^{-1}\right)$. However, no sign of toxicity or mutagenicity was found in mice. Finally, the antineoplastic effect of $L$. pacari was seen in human and murine lung cancer cells (Cordeiro et al., 2019). Lima et al. (2013), through the Ames test and the bone marrow micronucleus test in mice, discovered antigenotoxic and anticytotoxic properties of this plant. Although promising, further studies are needed to elucidate these species' anti-tumor activity; it is also necessary to investigate toxic adverse effects on human organisms to ensure safe use without risk to human health.

All versatile species cited for endocrine, nutritional, and metabolic diseases have reports of anti-diabetic activity in the literature. Pereira et al. (2015) report the anti-diabetic effect of $H$. speciosa by inhibiting $\alpha$-glucosidase and increased glucose uptake. Mishra et al. (2013) observed the hypoglycemic activity of $S$. dulcis by significantly inhibiting the blood glucose level in vivo tests. Morais et al. (2020) also found inhibition of enzymes related to type II diabetes ( $\alpha$-amylase and $\alpha$-glucosidase) by $\mathrm{H}$. drasticus. These results favor support for the traditional use of these species against diabetes mellitus. However, few studies still prove medicinal properties to guarantee the effective use of these plants against the disease.

Regarding infectious and parasitic diseases, there are scientific studies related to the antimicrobial activity of several species used in the popular treatment of infections, for example, the antibacterial activity of $A$. urundeuva against Staphylococcus aureus (ATCC 25923), Staphylococcus epidermidis (ATCC 12228), Escherichia coli (ATCC 25922), Pseudomonas aeruginosa (ATCC 27853) and Salmonella enteritidis (INCQS 500258) (Araújo et al., 2017); moderate antifungal activity of Scoparia dulcis against Aspergillus niger (NCIM No. 1055) and Candida albicans (NCIM N. 3471) (Parvataneni, 2019); and antiparasitic activity of L. ferrea in the inhibition of promastigote forms of Leishmania amazonensis and amastigotes of Leishmania guyanensis (Comandolli-Wyrepkowski et al., 2017).

The antimicrobial activities of these species and other important activities should be attributed mainly to their groups of chemical constituents. As shown in Table 2, several groups of secondary metabolites found in these medicinal plants were found, such as flavonoids and terpenoids, known for their important antioxidant, anti-inflammatory, antimutagenic, anticancer, antifungal, anti-viral, antibacterial, and antiparasitic properties (Cushnie \& Lamb, 2005; Duru \& Çayan, 2015; Panche et al., 2016).

\section{Conclusion}

The flora of Chapada of Araripe has a great wealth of medicinal species, but there is still a need for scientific study to prove the pharmacological effects of these species. This study provides a checklist on the ethnomedicinal uses of various plants with high therapeutic versatility, such as $C$. langsdorffii, L. pacari, $H$. stigonocarpa, A. urundeuva, and $H$. speciosa. It can support research to investigate new drugs and bioactive molecules. 
Finally, we emphasize that Chapada of Araripe is a region of great biological and cultural value, important for preserving local flora and traditional knowledge.

\section{Acknowledgments}

Authors thank the Regional University of Cariri (URCA) and the Multiprofessional Residency Program in Public Health (PRMS) for all the support during the development of this study.

\section{References}

Agra, M. D. F.; Baracho, G. S.; Nurit, K.; Basílio, I. J. L. D.; Coelho, V. P. M. 2007. Medicinal and poisonous diversity of the flora of "Cariri Paraibano", Brazil. Journal of Ethnopharmacology, 111, 383-395. DOI: 10.1016/j.jep.2006.12.007

Aguiar, L. C. G. G.; Barros, R. F. M. 2012. Plantas medicinais cultivadas em quintais de comunidades rurais no domínio do cerrado piauiense (Município de Demerval Lobão, Piauí, Brasil). Revista Brasileira de Plantas Medicinais, 14, 419-434. DOI: 10.1590/S1516-05722012000300001

Albuquerque, U. P. 2001. The use of medicinal plants by the cultural descendants of African people in Brazil. Acta Farmacéutica Bonaerense, 20, 139-144.

Albuquerque, U. P.; Medeiros, P. M.; Almeida, A. L. S.; Monteiro, J. M.; Lins-Neto, E. M. D. F.; Melo, J. G.; Santos, J. P. 2007a. Medicinal plants of the Caatinga (semi-arid) vegetation of NE Brazil: a quantitative approach. Journal of Ethnopharmacology, 114, 325-354. DOI: 10.1016/j.jep.2007.08.017

Albuquerque, U. P.; Monteiro, J. M.; Ramos, M. A.; Amorim, E. L. C. 2007b. Medicinal and magic plants from a public market in northeastern Brazil. Journal of Ethnopharmacology, 110, 76-91. DOI: 10.1016/j.jep.2006.09.010

Albuquerque, U. P.; Oliveira, R. F. 2007. Is the use-impact on native Caatinga species in Brazil reduced by the high species richness of medicinal plants?. Journal of Ethnopharmacology, 113, 156-170. DOI: 10.1016/j.jep.2007.05.025

Albuquerque, U. P.; Soldati, G. T.; Sieber, S. S.; Medeiros, P. M.; Sá, J. C.; Souza, L. C. 2011. Rapid ethnobotanical diagnosis of the Fulni-ô Indigenous lands (NE Brazil): floristic survey and local conservation priorities for medicinal plants. Environment, development and sustainability, 13, 277-292. DOI: 10.1007/s10668-010-9261-9
Alcantara, M. S.; Lucena, C. M.; Lucena, R. F. P.; Cruz, D. D. 2020. Ethnobotany and Management of Dimorphandra gardneriana in a Protected Area of Chapada do Araripe Semiarid Ceará, Northeastern Brazil. Environmental Management, 65, 420-432. DOI: 10.1007/s00267-020-01253-0

Alencar, N. L.; Araújo, T. A.S.; Amorim, E. L. C.; Albuquerque, U. P. 2010. The inclusion and selection of medicinal plants in traditional pharmacopoeias-evidence in support of the diversification hypothesis. Economic Botany, 64, 68-79. DOI: 10.1007/s12231-009-9104-5

Almeida, C. F. C. B. R.; Silva, T. D. L.; Amorim, E. L. C.; Maia, M. D. S.; Albuquerque, U. P. 2005. Life strategy and chemical composition as predictors of the selection of medicinal plants from the Caatinga (Northeast Brazil). Journal of arid environments, 62, 127-142. DOI: 10.1016/j.jaridenv.2004.09.020

Almeida, M. L. B.; Souza-Freitas, W. E.; Morais, P. L. D.; Sarmento, J. D. A.; Alves, R. E. 2016. Bioactive compounds and antioxidant potential fruit of Ximenia americana L. Food Chemistry, 192, 1078-1082. DOI: 10.1016/j.foodchem.2015.07.129

Almeida, S. C. X.; Silva, A.; Sousa, N. R. T.; Amorim, I. H. F.; Leite, B. G.; Neves, K. R. T.; Barros-Viana, G. S. 2019. Antinociceptive and anti-inflammatory activities of a triterpene-rich fraction from Himatanthus drasticus. Brazilian Journal of Medical and Biological Research, 52. DOI: 10.1590/1414431x20197798

Amaral, G. M.; Fontana, C.; Gasper, A. L.; Freitas, D. S.; Sevegnani, L. 2015. Aspectos da distribuição de Mimosoideae (Fabaceae) arbóreas no planalto de Santa Catarina, sul do Brasil. Neotropical Biology \& Conservation, 10, 74-84. DOI: 10.4013/nbc.2015.102.03

Anyaegbu, O. C.; Ajayi, A. M.; Adedapo, A. D. 2017. Hypolipidemic and antioxidant effects of the Methanolic stem bark extract of Anacardium occidentale Linn. in triton-X 100 induced hyperlipidemic rats. Oriental Pharmacy and Experimental Medicine, 17, 211-221. DOI: 10.1007/s13596-017-0262-1

Aponjolosun, S. B.; Fasola, R. T. 2020. Phytochemical, Antimicrobial and Toxicity Evaluation of Anacardium occidentale Linn. Leaf Extracts. Tropical Journal of Natural Product Research, 4, 113-122. DOI: 10.26538/tjnpr/v4i4.1

Aragão, T. P.; Prazeres, L. D. K. T. D.; Brito, S. A.; Neto, P. J. R.; Rolim, L. A.; Almeida, J. R. G. D. S.; Wanderley, A. G. 2018. Contribution of secondary metabolites to the 
gastroprotective effect of aqueous extract of Ximenia americana L. (Olacaceae) stem bark in rats. Molecules, 23, 112. DOI: 10.3390/molecules 23010112

Araújo, Í. D. R.; Aquino, N. C.; Aguiar-Guerra, A. C. V.; Almeida-Júnior, R. F.; Araújo, R. M.; Araújo Júnior, R. F.; Andrade, V. S. 2017. Chemical composition and evaluation of the antibacterial and Cytotoxic activities of the essential oil from the leaves of Myracrodruon urundeuva. BMC complementary and alternative medicine, 17, 1-8. DOI: 10.1186/s12906-017-1918-6

Assumpção, C. F.; Bachiega, P.; Morzelle, M. C.; Nelson, D. L.; Ndiaye, E. A.; Rios, A. D. O.; Souza, E. C. D. 2014. Characterization, antioxidant potential and cytotoxic study of mangaba fruits. Ciência Rural, 44, 12971303. DOI: $10.1590 / 0103-8478$ cr20130855

Baldauf, C.; Santos, F. A. M. 2013. Ethnobotany, traditional knowledge, and diachronic changes in non-timber forest products management: a case study of Himatanthus drasticus (Apocynaceae) in the Brazilian Savanna. Economic Botany, 67, 110-120. DOI: $10.1007 / \mathrm{s} 12231-013-9228-5$

Bastos, F. D. H.; Cordeiro, A. M. N.; Macedo, F. E.; Azevedo, R. E. S. D. 2016. A gestão ambiental nas paisagens da bacia do Araripe no Estado do Ceará. Confins. Revista francobrasileira de geografia, 29. DOI: 10.4000/confins. 11509

Batista, W. V. S. M.; Pôrto, K. C.; Santos, N. D. D. 2018. Distribution, ecology, and reproduction of bryophytes in a humid enclave in the semiarid region of northeastern Brazil. Acta Botanica Brasilica, 32, 303-313. DOI: 10.1590/0102-33062017abb0339

Bennett, B. C.; Prance, G. T. 2000. Introduced plants in the indigenous pharmacopoeia of Northern South America. Economic Botany, 54, 90-102. DOI: 10.1007/BF02866603

Bezerra, J. W. A.; da Silva, M. A. P.; Batista, A. P.; Mendonça, A. C. A. M.; Oliveira-Sousa, J. F.; Santos, M. A. F.; Silva, V. B. 2018. Biometria e quebra de dormência em Dimorphandra gardneriana Tul. (Faveira) (Fabaceae). Revista Cubana de Plantas Medicinales, 23.

Bieski, I. G. C.; Leonti, M.; Arnason, J. T.; Ferrier, J.; Rapinski, M.; Violante, I. M. P.; Silva, D. R. 2015. Ethnobotanical study of medicinal plants by population of valley of Juruena region, legal amazon, Mato Grosso, Brazil. Journal of Ethnopharmacology, 173, 383423. DOI: 10.1016/j.jep.2015.07.025

Bieski, I. G. C.; Rios-Santos, F.; Oliveira, R. M.; Espinosa, M. M.; Macedo, M.; Albuquerque,
U. P.; Oliveira-Martins, D. T. 2012. Ethnopharmacology of medicinal plants of the Pantanal region (Mato Grosso, Brazil). Evidence-Based Complementary and Alternative Medicine, 2012. DOI: 10.1155/2012/272749

Bitu, V. D. C. N.; Bitu, V. D. C. N.; Matias, E. F. F.; Lima, W. P.; Portelo, A. C.; Coutinho, H. D. M.; Menezes, I. R. A. 2015. Ethnopharmacological study of plants sold for therapeutic purposes in public markets in Northeast Brazil. Journal of Ethnopharmacology, 172, 265-272. DOI: 10.1016/j.jep.2015.06.022

Bolson, M.; Hefler, S. R.; Dall, E. I.; Chaves, O.; Gasparroto-Junior, A.; Cardozo-Junior, E. L. 2015. Ethno-medicinal study of plants used for treatment of human ailments, with residents of the surrounding region of forest fragments of Paraná, Brazil. Journal of Ethnopharmacology, 161, 1-10. DOI: 10.1016/j.jep.2014.11.045

Carvalho, C. E. S.; Sobrinho-Junior, E. P. C.; Brito, L. M.; Nicolau, L. A. D.; Carvalho, T. P.; Moura, A. K. S.; Carvalho, F. A. A. 2017. Anti-Leishmania activity of essential oil of Myracrodruon urundeuva (Engl.) Fr. All.: Composition, cytotoxity and possible mechanisms of action. Experimental Parasitology, 175, 59-67. DOI: 10.1016/j.exppara.2017.02.012

Castro, C. B.; Luz, L. R.; Guedes, J. A.; Porto, D. D.; Silva, M. F. S.; Silva, G. S.; Pessoa, C. Ó. 2020. Metabolomics-Based Discovery of Biomarkers with Cytotoxic Potential in Extracts of Myracrodruon urundeuva. Journal of the Brazilian Chemical Society, 31, 775-787. DOI:10.21577/01035053.20190242

Chaibub, B. A.; Martins, J. L.; Paula, J. R.; Santos, S. D. C.; Costa, E. A.; Bara, M. T. F. 2020. Gastroprotective activity of punicalagin and Lafoensia pacari in mice. Rev. Bras. Farmacogn, 30, 423-426. DOI: 10.1007/s43450-020-00007-7

Colares, A.; Cordeiro, L.; Costa, J. G. M.; Silveira, E.; Campos, A.; Cardoso, A. 2008. Phytochemical and biological preliminary study of Himatanthus drasticus (Mart.) Plumel (Janaguba). Pharmacognosy Magazine, 4, 73-77.

Comandolli-Wyrepkowski, C. D.; Jensen, B. B.; Grafova, I.; Santos, P. A. D.; Barros, A. M. C.; Soares, F. V.; Franco, A. M. R. 2017. Antileishmanial activity of extracts from Libidibia ferrea: development of in vitro and in vivo tests. Acta Amazonica, 47, 331-340. DOI: $10.1590 / 1809-4392201700871$ 
Cordeiro, Y. D. G.; Rochetti, A. L.; Souza, V. C.; Silva, E. R. D.; Scatolini, A. M.; Genovese, M. I.; Fukumasu, H. 2019. Antineoplastic Effect of Procyanidin-rich Extract of Lafoensia Pacari in Lung Carcinoma Cells. Brazilian Archives of Biology and Technology, 62. DOI: 10.1590/1678-43242019160638

Costa, A. R.; Lima-Silva, J. R.; Oliveira, T. J. S.; Silva, T. G.; Pereira, P. S.; Oliveira-Borba, E. F.; Menezes, I. R. A. 2020. Phytochemical profile of Anacardium occidentale L. (cashew tree) and the cytotoxic and toxicological evaluation of its bark and leaf extracts. South African Journal of Botany, 135, 355-364. DOI: 10.1016/j.sajb.2020.09.017

Cushnie, T. T.; Lamb, A. J. 2005. Antimicrobial activity of flavonoids. International journal of antimicrobial agents, 26, 343-356. DOI: 10.1016/j.ijantimicag.2005.09.002

Davuluri, T.; Chennuru, S.; Pathipati, M.; Krovvidi, S.; Rao, G. S. 2019. In vitro anthelmintic activity of three tropical plant extracts on Haemonchus contortus. Acta Parasitologica, 65, 1-8. DOI: 10.2478/s11686-019-00116-X

Dimech, G. S.; Soares, L. A. L.; Ferreira, M. A.; Oliveira, A. G. V.; Carvalho, M. D. C.; Ximenes, E. A. 2013. Phytochemical and antibacterial investigations of the extracts and fractions from the stem bark of Hymenaea stigonocarpa Mart. ex Hayne and effect on ultrastructure of Staphylococcus aureus induced by hydroalcoholic extract. The Scientific World Journal, 8.

Dorigoni, P. A.; Ghedini, P. C.; Fróes, L. F.; Baptista, K. C.; Ethur, A. B. M.; Baldisserotto, B.; Záchia, R. A. 2001. Levantamento de dados sobre plantas medicinais de uso popular no município de São João do Polesine, RS. II-Relação entre enfermidades e espécies estudadas. Revista Brasileira de Plantas Medicinais. Botucatu, 1, 69-79.

Duru, M. E.; Çayan, G. T. 2015. Biologically active terpenoids from mushroom origin: a review. Records of Natural Products, 9, 456.

Falcão, T. R.; Araújo, A. A. D.; Soares, L. A. L.; Farias, I. B. D.; Silva, W. A. V. D.; Ferreira, M. R. A.; Guerra, G. C. B. 2019. Libidibia ferrea fruit crude extract and fractions show anti-inflammatory, antioxidant, and antinociceptive effect in vivo and increase cell viability in vitro. Evidence-Based Complementary and Alternative Medicine, 2019, 14. DOI: $10.1155 / 2019 / 6064805$
Figueredo, F. G.; Lucena, B. F.; Tintino, S. R.; Matias, E. F.; Leite, N. F.; Andrade, J. C.; Rodrigues, F. F. 2014. Chemical composition and evaluation of modulatory of the antibiotic activity from extract and essential oil of Myracrodruon urundeuva. Pharmaceutical biology, 52, 560-565. DOI: 10.3109/13880209.2013.853810

Floriano, J. F.; Capuano-Neto, F.; Mota, L. S. L. S.; Furtado, E. L.; Ferreira, R. S.; Barraviera, B.; Oliveira-Graeff, C. F. 2016. Comparative study of bone tissue accelerated regeneration by latex membranes from Hevea brasiliensis and Hancornia speciosa. Biomedical Physics \& Engineering Express, 2, 045007. DOI: 10.1088/2057-1976/2/4/045007

Frausin, G.; de Freitas-Hidalgo, A.; Lima, R. B. S.; Kinupp, V. F.; Ming, L. C.; Pohlit, A. M.; Milliken, W. 2015. An ethnobotanical study of anti-malarial plants among indigenous people on the upper Negro River in the Brazilian Amazon. Journal of Ethnopharmacology, 174, 238-252. DOI: 10.1016/j.jep.2015.07.033

Galdino, P. M.; Carvalho, A. A.; Florentino, I. F.; Martins, J. L.; Gazola, A. C.; Paula, J. R.; Lima, T. C. M. 2015. Involvement of monoaminergic systems in the antidepressant-like properties of Lafoensia pacari A. St. Hil. Journal of Ethnopharmacology, 170, 218-225. DOI: 10.1016/j.jep.2015.05.015

Galvão, W. A.; Braz-Filho, R.; Canuto, K. M.; Ribeiro, P. R. V.; Campos, A. R.; Moreira, A. C. O. M.; Gonçalves, N. G. G. 2018. Gastroprotective and anti-inflammatory activities integrated to chemical composition of Myracrodruon urundeuva Allemão-A conservationist proposal for the species. Journal of Ethnopharmacology, 222, 177189. DOI: $10.1016 /$ j.jep.2018.04.024

Garcez, F. R.; Garcez, W. S.; Yoshida, N. C.; Figueiredo, P. O. 2016. A diversidade dos constituintes químicos da flora de Mato Grosso do Sul e sua relevância como fonte de substâncias bioativas. Revista Virtual de Química, 8, 97-129. DOI: 10.5935/19846835.20160008

Gelmini, F.; Beretta, G.; Anselmi, C.; Centini, M.; Magni, P.; Ruscica, M.; Facino, R. M. 2013. GC-MS profiling of the phytochemical constituents of the oleoresin from Copaifera langsdorffii Desf. and a preliminary in vivo evaluation of its antipsoriatic effect. International Journal of Pharmaceutics, 440, 170-178.DOI:10.1016/j.ijpharm.2012.08.021 
Guimarães, B. O.; Oliveira, A. P.; Morais, I. L. 2019. Plantas Medicinais de Uso Popular na Comunidade Quilombola de Piracanjuba-Ana Laura, Piracanjuba, GO. Fronteiras: Journal of Social, Technological and Environmental Science, $\quad 8$. DOI: $10.21664 / 2238$ 8869.2019v8i3.p196-220

Gushiken, L. F. S.; Hussni, C. A.; Bastos, J. K.; Rozza, A. L.; Beserra, F. P.; Vieira, A. J.; Nóbrega, R. H. 2017. Skin wound healing potential and mechanisms of the hydroalcoholic extract of leaves and oleoresin of Copaifera langsdorffii Desf. Kuntze in rats. Evidence-Based Complementary and Alternative Medicine, 2017, 16. DOI: 10.1155/2017/6589270

IBAMA-Instituto Brasileiro do Meio Ambiente e dos Recursos Naturais Renováveis. 2004. Plano de manejo da Floresta Nacional do Araripe. Brasília-DF, 318.

Sousa-Júnior, J. R..; Collevatti, R. G.; Lins-Neto, E. M. F.; Peroni, N.; Albuquerque, U. P. 2018. Traditional management affects the phenotypic diversity of fruits with economic and cultural importance in the Brazilian Savanna. Agroforestry Systems, 92, 11-21. DOI: 10.1007/s10457-016-0005-1

Pereira-Júnior, L. R.; Andrade, A. P. D.; Araújo, K. D.; Barbosa, A. D. S.; Barbosa, F. M. 2014. Espécies da Caatinga como alternativa para o desenvolvimento de novos fitofármacos. Floresta e Ambiente, 21, 509-520. DOI: 10.1590/2179-8087.024212

Ferreira-Júnior, W. S. F.; Santoro, F. R.; Nascimento, A. L. B.; Avilez, W. M. T.; Zank, S.; Silva, N. F.; Albuquerque, U. P.; Araujo, E. L. 2015. Checklist das plantas medicinais na chapada do Araripe. In: Abuquerque, U. P.; Meiado, M. V. Sociobiodiversidade na Chapada do Araripe, Recife, PE: NUPPEA, 1, 431-450.

Kobayashi, Y. T. S.; de Almeida, V. T.; Bandeira, T.; de Alcantâra, B. N.; da Silva, A. S. B.; Barbosa, W. L. R.; Almeida, M. B. 2015. Avaliação fitoquímica e potencial cicatrizante do extrato etanólico dos frutos de Jucá (Libidibia ferrea) em ratos Wistar. Brazilian Journal of Veterinary Research and Animal Science, $\quad 52, \quad 34-40 . \quad$ DOI: 10.11606/issn.1678-4456.v52i1p34-40

Lemos, I. C. S.; Araújo Delmondes, G.; Santos, A. D. F.; Santos, E. S.; Oliveira, D. R.; Figueiredo, P. R. L.; Kerntopf, M. R. 2016. Ethnobiological survey of plants and animals used for the treatment of acute respiratory infections in children of a traditional community in the municipality of Barbalha,
Ceará, Brazil. African Journal of Traditional, Complementary and Alternative Medicines, 13, 166-175.

Lemos, M.; Santin, J. R.; Mizuno, C. S.; Boeing, T.; Sousa, J. P. B. D.; Nanayakkara, D.; Andrade, S. F. D. 2015. Copaifera langsdorffii: evaluation of potential gastroprotective of extract and isolated compounds obtained from leaves. Revista Brasileira de Farmacognosia, 25, 238-245. DOI: 10.1016/j.bjp.2015.05.005

Lima, D. C. S.; Silva, C. R.; Sampaio, B. L.; Paula, J. R. D.; Lee, C. C. 2013. Antigenotoxic, and anticytotoxic activities of an ethanolic extract of Lafoensia pacari (Lythraceae) stem bark in bacteria and mice. Molecular Research, 12, 3887-3896.

Lima, J. P.; Azevedo, L.; Souza, N. J.; Nunes, E. E.; Boas, E. V. D. B. V. 2015. First evaluation of the antimutagenic effect of mangaba fruit in vivo and its phenolic profile identification. Food Research International, 75, 216-224. DOI: 10.1016/j.foodres.2015.05.045

Loiola, M. I. B.; Araújo, F. S.; Lima-Verde, L. W.; Souza, S. S. G.; Matias, L. Q.; Menezes, M. O. T.; Oliveira, S. F. 2015. Flora da Chapada do Araripe. In: Abuquerque, U. P.; Meiado, M. V. Sociobiodiversidade na Chapada do Araripe, Recife, PE: NUPPEA, 1, pp. 103148.

Luna, M. S. M.; Paula, R. A.; Costa, R. B.; Anjos, J. V.; Silva, M. V.; Correia, M. T. S. 2020. Bioprospection of Libidibia ferrea var. ferrea: Phytochemical properties and antibacterial activity. South African Journal of Botany, 130, 103-108. DOI: 10.1016/j.sajb.2019.12.013

Macêdo, D. G.; Ribeiro, D. A.; Coutinho, H. D.; Menezes, I. R.; Souza, M. M. 2015. Práticas terapêuticas tradicionais: uso e conhecimento de plantas do cerrado no estado de Pernambuco (Nordeste do Brasil). Boletín Latinoamericano y del Caribe de Plantas Medicinales y Aromáticas, 14, 491-508.

Macêdo, M. J. F.; Ribeiro, D. A.; Oliveira-Santos, M.; Macedo, D. G.; Macedo, J. G. F.; Almeida, B. V.; Almeida-Souza, M. M. 2018. Fabaceae medicinal flora with therapeutic potential in Savanna areas in the Chapada do Araripe, Northeastern Brazil. Revista Brasileira de Farmacognosia, 28, 738-750. DOI: 10.1016/j.bjp.2018.06.010

Magno-Silva, E. R.; Rocha, T. T.; TavaresMartins, A. C. C. 2020. Ethnobotany and Ethnopharmacology of medicinal plants used in communities of the Soure Marine Extractive Reserve, Pará State, Brazil. 
Boletín Latinoamericano y del Caribe de Plantas Medicinales y Aromáticas, 19.

Maranhão, C. A.; Pinheiro, I. O.; Santana, A. L.; Oliveira, L. S.; Nascimento, M. S.; Bieber, L. W. 2013. Antitermitic and antioxidant activities of heartwood extracts and main flavonoids of Hymenaea stigonocarpa Mart. International Biodeterioration \& Biodegradation, $79, \quad 9-13 . \quad$ DOI: 10.1016/j.ibiod.2013.01.005

Martínez-Aguilar, Y.; Rodríguez, F. S.; Saavedra, M. A.; Espinosa, R., H.; Yero, O. M. 2012. Metabolitos secundarios y actividad antibacteriana in vitro de extractos de hojas de Anacardium occidentale L.(marañón). Revista Cubana de Plantas Medicinales, 17, 320-329.

Menezes, I. R. A.; Costa, R. H. S.; Boligon, A. A.; Rolón, M.; Coronel, C.; Vega, C.; Albuquerque, T. R. 2019. Ximenia americana L. enhances the antibiotic activity and inhibit the development of kinetoplastid parasites. Comparative immunology, microbiology and infectious diseases, 64, 40-46. DOI: 10.1016/j.cimid.2019.02.007

Mesquita, U. O.; Tavares-Martins, A. C. C. 2018. Etnobotánica de plantas medicinales en la comunidad de Caruarú, Isla del Mosqueiro, Belém-PA, Brasil. Boletín latinoamericano y del Caribe de plantas medicinales y aromáticas, 17, 130-159.

Miguéis, G. D. S.; Silva, R. H.; Damasceno-Júnior, G. A.; Guarim-Neto, G. 2019. Plants used by the rural community of Bananal, Mato Grosso, Brazil: Aspects of popular knowledge. PloS One, 14, 0210488. DOI: 10.1371/journal.pone.0210488

Mishra, M. R.; Mishra, A.; Pradhan, D. K.; Panda, A. K.; Behera, R. K.; Jha, S. 2013. Antidiabetic and antioxidant activity of Scoparia dulcis Linn. Indian Journal of Pharmaceutical Sciences, 75, 610-614.

Morais, F. S.; Canuto, K. M.; Ribeiro, P. R.; Silva, A. B.; Pessoa, O. D.; Freitas, C. D.; Ramos, M. V. 2020. Chemical profiling of secondary metabolites from Himatanthus drasticus (Mart.) Plumel latex with inhibitory action against the enzymes $\alpha$-amylase and $\alpha$ glucosidase: In vitro and in silico assays. Journal of Ethnopharmacology, 253, 112644. DOI: 10.1016/j.jep.2020.112644

Moro, M. F.; Macedo, M. B.; Moura-Fé, M. M. D.; Castro, A. S. F.; Costa, R. C. D. 2015. Vegetação, unidades fitoecológicas e diversidade paisagística do estado do Ceará. Rodriguésia, 66, 717-743. DOI: $10.1590 / 2175-7860201566305$
Moura, D. F.; Rocha, T. A.; Melo-Barros, D.; Silva, M. M.; Lira, M. A. D. C.; SantosSouza, T. G.; Souza, I. A. 2020. Evaluation of the cytotoxicity, oral toxicity, genotoxicity, and mutagenicity of the latex extracted from Himatanthus drasticus (Mart.) Plumel (Apocynaceae). Journal of Ethnopharmacology, 253, 112567. DOI: 10.1016/j.jep.2020.112567

Mustapha, A. A.; Owuna, G.; Ogaji, J. O.; Ishaq, U. I.; Idris, M. M. 2015. Phytochemical screening and inhibitory activities of anacardium occidentale leave extracts against some clinically important bacterial isolates. Int J Pharmaco Phytochem Res, 7, 365-369.

Muthumani, P.; Chiristina, A. J. M.; Venkataraman, S.; Meera, R.; Abraham, J.; Devi, P. 2016. Preliminary phytochemical screening, chemical investigation, enzyme inhibiting activity and atomic absorption spectrophotometric determination of minerals of plant extracts of Scoparia dulcis. LINN. International Journal of Pharmaceutical Sciences Review and Research, 2,16.

Negrelle, R. R. B.; Fornazzari, K. R. 2007. Ethnobotanical study in two rural communities (Limeira and Riberião Grande) in Guaratuba (Paraná, Brazil). Revista Brasileira de Plantas Medicinais, 9, 36-54.

Novaes, R. L. M.; Laurindo, R. D. S. 2014. Morcegos da Chapada do Araripe, nordeste do Brasil. Papéis avulsos de Zoologia, 54, 315-328.

Nur-e-Alam, M.; Ahmed, S.; Yousaf, M.; Khan, S. I.; Mothana, R. A.; Al-Rehaily, A. J. 2020. Isolation and characterization of cytotoxic and anti-inflammatory constituents from Scoparia dulcis L. Journal of Chemical Research, 44.4 DOI: 10.1177/1747519819901100

Oliveira, S. G. D.; Moura, F. R. R.; Demarco, F. F.; Silva-Nascente, P.; Del-Pino, F. A. B.; Lund, R. G. 2012. An ethnomedicinal survey on phytotherapy with professionals and patients from Basic Care Units in the Brazilian Unified Health System. Journal of Ethnopharmacology, 140, 428-437. DOI: 10.1016/j.jep.2012.01.054

Orsi, P. R.; Bonamin, F.; Severi, J. A.; Santos, R. C.; Vilegas, W.; Hiruma-Lima, C. A.; Di Stasi, L. C. 2012. Hymenaea stigonocarpa Mart. ex Hayne: A Brazilian medicinal plant with gastric and duodenal anti-ulcer and antidiarrheal effects in experimental rodent models. Journal of Ethnopharmacology, 143, 81-90. DOI: 10.1016/j.jep.2012.06.001 
Orsi, P. R.; Seito, L. N.; Di Stasi, L. C. 2014. Hymenaea stigonocarpa Mart. ex Hayne: A tropical medicinal plant with intestinal antiinflammatory activity in TNBS model of intestinal inflammation in rats. Journal of Ethnopharmacology, 151, 380-385. DOI; 10.1016/j.jep.2013.10.056

Pagani, E., F. L.; Santos, J.; Rodrigues, E. 2017. Culture-Bound Syndromes of a Brazilian Amazon Riverine population: Tentative correspondence between traditional and conventional medicine terms and possible ethnopharmacological implications. Journal of Ethnopharmacology, 203, 80-89. DOI: 10.1016/j.jep.2017.03.024

Panche, A. N.; Diwan, A. D.; Chandra, S. R. 2016. Flavonoids: an overview. Journal of Nutritional Science, 5, E47. DOI: 10.1017/jns.2016.41

Parvataneni, R. 2020. Biogenic synthesis and characterization of silver nanoparticles using aqueous leaf extract of Scoparia dulcis L. and assessment of their antimicrobial property. Drug and Chemical Toxicology, 43, 307-321. DOI: $10.1080 / 01480545.2018 .1505903$

Penido, A. B.; Morais, S. M. D.; Ribeiro, A. B.; Silva, A. Z. 2016. Ethnobotanical study of medicinal plants in Imperatriz, State of Maranhão, Northeastern Brazil. Acta Amazonica, 46, 345-354. DOI: $10.1590 / 1809-4392201600584$

Pereira, A. C.; Pereira, A. B. D.; Moreira, C. C.; Botion, L. M.; Lemos, V. S.; Braga, F. C.; Cortes, S. F. 2015. Hancornia speciosa Gomes (Apocynaceae) as a potential antidiabetic drug. Journal of Ethnopharmacology, 161, 30-35. DOI: 10.1016/j.jep.2014.11.050

Pereira, F. J.; Martins, F. T.; Corrêa, R. S.; Moreira, M. E.; Costa, A. M. D. D.; Santos, M. H.; Barbosa, L. C. 2008. Isolamento, composição química e atividade anti-inflamatória do óleo essencial do pericarpo de Copaifera langsdorffii Desf. de acordo com hidrodestilações sucessivas. Lat. Am. J. Pharm, 27, 369-74.

Pereira, L. O. M.; Vilegas, W.; Tangerina, M. M. P.; Arunachalam, K.; Balogun, S. O.; Orlandi-Mattos, P. E.; Martins, D. T. O. 2018. Lafoensia pacari A. St.-Hil.: wound healing activity and mechanism of action of standardized hydroethanolic leaves extract. Journal of Ethnopharmacology, 219, 337350. DOI: 10.1016/j.jep.2018.02.038

Pio, I. D. S. L.; Lavor, A. L.; Damasceno, C. M. D.; Menezes, P. M. N.; Silva, F. S.; Maia, G. L. A. 2019. Traditional knowledge and uses of medicinal plants by the inhabitants of the islands of the São Francisco River, Brazil and preliminary analysis of Rhaphiodon echinus (Lamiaceae). Brazilian Journal of Biology, 79, 87-99. DOI: 10.1590/1519-6984.177447

Ribeiro, D. A.; Macedo, D. G.; Oliveira, L. G. S.; Santos, M. O.; Almeida, B. V.; Macedo, J. G. F.; Souza, M. M. A. 2017a. Conservation priorities for medicinal woody species in a cerrado area in the Chapada do Araripe, northeastern Brazil. Environment, Development and Sustainability, 21, 61-77. DOI: 10.1007/s10668-017-0023-9

Ribeiro, D. A.; Oliveira, L. G. S.; Macêdo, D. G.; Menezes, I. R. A.; Costa, J. G. M.; Silva, M. A. P.; Almeida-Souza, M. M. 2014. Promising medicinal plants for bioprospection in a Cerrado area of Chapada do Araripe, Northeastern Brazil. Journal of Ethnopharmacology, 155, 1522-1533. DOI: 10.1016/j.jep.2014.07.042

Ribeiro, R. V.; Bieski, I. G. C.; Balogun, S. O.; Oliveira-Martins, D. T. 2017b. Ethnobotanical study of medicinal plants used by Ribeirinhos in the North Araguaia microregion, Mato Grosso, Brazil. Journal of Ethnopharmacology, 205, 69-102. DOI: 10.1016/j.jep.2017.04.023

Rodrigues, V. E. G.; Carvalho, D. D. 2001. Levantamento etnobotânico de plantas medicinais no domínio do cerrado na região do Alto Rio Grande-Minas Gerais. Ciência e Agrotecnologia, 25, 102-123.

Santana, B. F.; Voeks, R. A.; Funch, L. S. 2016. Ethnomedicinal survey of a maroon community in Brazil's Atlantic tropical forest. Journal of Ethnopharmacology, 181, 37-49. DOI: $10.1016 /$ j.jep.2016.01.014

Santos, E. B.; Dantas, G. S.; Santos, H. B.; Diniz, M. F.; Sampaio, F. C. 2009. Estudo etnobotânico de plantas medicinais para problemas bucais no município de João Pessoa, Brasil. Revista Brasileira de Farmacognosia, 19, 321-324. DOI: 10.1590/S0102-695X2009000200024

Santos, J. C. B.; Castro-Junior, J. A. D. A.; Wakui, V. G.; Rodrigues, J. F.; Arruda, M. O.; Monteiro, A. D. S.; Grisotto, M. A. G. 2017. Himatanthus drasticus leaves: chemical characterization and evaluation of their antimicrobial, antibiofilm, antiproliferative activities. Molecules, 22, 910. DOI: 10.3390/molecules 22060910

Santos, J. C.; Leal, I. R.; Almeida-Cortez, J. S.; Fernandes, G. W.; Tabarelli, M. 2011. Caatinga: the scientific negligence experienced by a dry tropical forest. Tropical 
Conservation Science, 4, 276-286. DOI: 10.1177/194008291100400306

Santos, U. P.; Campos, J. F.; Torquato, H. F. V.; Paredes-Gamero, E. J.; Carollo, C. A.; Estevinho, L. M.; Santos, E. L. 2016. Antioxidant, antimicrobial and cytotoxic properties as well as the phenolic content of the extract from Hancornia speciosa Gomes. PLoS One, 11, e0167531. DOI: 10.1371/journal.pone.0167531

Saraiva, M. E.; Alencar-Ulisses, A. V. R.; Ribeiro, D. A.; Oliveira, L. G. S.; Macêdo, D. G.; Sousa, F. D. F. S.; Almeida-Souza, M. M. 2015. Plant species as a therapeutic resource in areas of the savanna in the state of Pernambuco, Northeast Brazil. Journal of Ethnopharmacology, 171, 141-153. DOI: 10.1016/j.jep.2015.05.034

Shettar, A. K.; Vedamurthy, A. B. 2017. Evaluation of in-vitro Anthelmintic Activity of Ximenia americana, Hopea ponga and Vitex leucoxylon. Pharmacognosy Journal, 9, 367-371. DOI: 10.5530/pj.2017.3.62

Silva, B. A. F.; Costa, R. H. S.; Fernandes, C. N.; Leite, L. H. I.; Ribeiro-Filho, J.; Garcia, T. R.; Menezes, I. R. A. 2018. HPLC profile and antiedematogenic activity of Ximenia americana L. (Olacaceae) in mice models of skin inflammation. Food and Chemical Toxicology, 119, 199-205. DOI: 10.1016/j.fct.2018.04.041

Silva, C. P. D.; Sousa, M. S. B.; Siguemoto, E. S.; Soares, R. A. M.; Arêas, J. A. G. 2014a. Chemical composition and antioxidant activity of jatobá-do-cerrado (Hymenaea stigonocarpa Mart.) flour. Food Science and Technology, 34, 597-603. DOI: 10.1590/1678-457x.6405

Silva, F. S.; Albuquerque, U. P.; Costa-Júnior, L. M.; Silva-Lima, A.; Nascimento, A. L. B.; Monteiro, J. M. 2014b. An ethnopharmacological assessment of the use of plants against parasitic diseases in humans and animals. Journal of Ethnopharmacology, 155, 1332-1341. DOI: 10.1016/j.jep.2014.07.036

Silva, J. G.; Grandi, A.; Almeida-Caetano, R.; Santos-Rodrigues, L.; Carnaúba, A. F.; Santos, A. M. S.; Silva, H. C. H. 2020. Are medicinal plants an alternative to the use of synthetic pharmaceuticals in animal healthcare in the Brazilian semi-arid?. Ethnobotany Research and Applications, 19, 1-20. DOI: 10.32859/era.19.02.1-20

Silva, M. I.; Sousa, F. C.; Gondim, A. P. 2005. Herbal therapy in primary health care in Maracanaú, Ceará, Brazil. Annals of
Pharmacotherapy, 39, 1336-1341. DOI: 10.1345/aph.1E178

Silva, V. D.; Nascimento, V. D.; Soldati, G. T.; Medeiros, M. F. T.; Albuquerque, U. D. 2010. Técnicas para análise de dados etnobiológicos. In: Albuquerque, U. P.; Lucena, R. F. P.; Cunha, L. V. F. C. Métodos e técnicas na pesquisa etnobiológica e etnoecológica. Recife, PE: NUPPEA, 187206.

Silva-Leite, K. E.; Assreuy, A. M.; Mendonça, L. F.; Damasceno, L. E.; Queiroz, M. G.; Mourão, P. A.; Pereira, M. G. 2017. Polysaccharide rich fractions from barks of Ximenia americana inhibit peripheral inflammatory nociception in mice: Antinociceptive effect of Ximenia americana polysaccharide rich fractions. Revista Brasileira de Farmacognosia, 27, 339-345. DOI: 10.1016/j.bjp.2016.12.001

Soares, F. P.; Fraga, A. F.; Neves, J. P. O.; Romero, N. R.; Bandeira, M. A. M. 2015. Estudo etnofarmacológico e etnobotânico de Himatanthus drasticus (Mart.) Plumel (janaguba). Revista Brasileira de Plantas Medicinais, $17, \quad 900-908 . \quad$ DOI: 10.1590/1983-084X/14_086

Sobeh, M.; Mahmoud, M. F.; Abdelfattah, M. A.; El-Beshbishy, H. A.; El-Shazly, A. M.; Wink, M. 2017. Hepatoprotective and hypoglycemic effects of a tannin rich extract from Ximenia americana var. caffra root. Phytomedicine, 33, 36-42. DOI: 10.1016/j.phymed.2017.07.003

Sousa, E. L. D.; Grangeiro, A. R. S.; Bastos, I. V. G. A.; Rodrigues, G. C. R.; Anjos, F. B. R. D.; Souza, I. A. D.; Sousa, C. E. L. D. 2010. Antitumor activity of leaves of Himatanthus drasticus (Mart.) Plumel-Apocynaceae (Janaguba) in the treatment of Sarcoma 180 tumor. Brazilian Journal of Pharmaceutical Sciences, 46, 199-203. DOI: 10.1590/S198482502010000200005

Souza, C. D. D.; Felfili, J. M. 2006. Uso de plantas medicinais na região de Alto Paraíso de Goiás, GO, Brasil. Acta Botanica Brasilica, 20, $135142 . \quad$ DOI: 10.1590/S010233062006000100013

Souza, R. K. D.; Silva, M. A. P.; Menezes, I. R. A.; Ribeiro, D. A.; Bezerra, L. R.; AlmeidaSouza, M. M. 2014. Ethnopharmacology of medicinal plants of carrasco, northeastern Brazil. Journal of Ethnopharmacology, 157, 99-104. DOI: 10.1016/j.jep.2014.09.001

Tamashiro-Filho, P.; Olaitan, B. S.; Almeida, D. A. T.; Silva-Lima, J. C.; Marson-Ascêncio, P. G.; Ascêncio, S. D.; Oliveira-Martins, D. T. 
2012. Evaluation of antiulcer activity and mechanism of action of methanol stem bark extract of Lafoensia pacari A. St.-Hil. (Lytraceae) in experimental animals. Journal of Ethnopharmacology, 144, 497-505. DOI: 10.1016/j.jep.2012.09.019

Ustulin, M.; Figueiredo, B. D. B.; Tremea, C.; Pott, A.; Pott, V. J.; Bueno, N. R.; Castilho, R. O. 2009. Plantas medicinais comercializadas no mercado municipal de Campo Grande-MS. Revista Brasileira de Farmacognosia, 19, 805-813. DOI: $\quad 10.1590 / \mathrm{S} 0102-$ 695X2009000500026

Vasconcelos, M. S.; Gomes-Rochette, N. F.; Oliveira, M. L. M.; Nunes-Pinheiro, D. C. S.; Tomé, A. R.; Maia-Sousa, F. Y.; Melo, D. F. 2015. Anti-inflammatory and wound healing potential of cashew apple juice (Anacardium occidentale L.) in mice. Experimental Biology and Medicine, 240, 1648-1655. DOI: 10.1177/1535370215576299

Vásquez, S. P. F.; Mendonça, M. S. D.; Noda, S. D. N. 2014. Etnobotânica de plantas medicinais em comunidades ribeirinhas do Município de Manacapuru, Amazonas,
Brasil. Acta Amazonica, 44, 457-472. DOI: 10.1590/1809-4392201400423

Vieira, L. S.; Sousa, R. S.; Lemos, J. R. 2015. Plantas medicinais conhecidas por especialistas locais de uma comunidade rural maranhense. Revista Brasileira de Plantas Medicinais, 17, 1061-1068. DOI: 10.1590/1983-084x/15_009

World Health Organization. 2019. International statistical classification of diseases and related health problems. $10^{\text {th }}$ revision.https://www.who.int/classifications/ icd/en

World Health Organization. 2018. Traditional and complementary medicine in primary health care (No. WHO/HIS/SDS/2018.37). World Health Organization.

Yazbek, P. B.; Matta, P.; Passero, L. F.; Santos, G.; Braga, S.; Assunção, L.; Barreto, E. H. P. 2019. Plants utilized as medicines by residents of Quilombo da Fazenda, Núcleo Picinguaba, Ubatuba, São Paulo, Brazil: A participatory survey. Journal of Ethnopharmacology, 244, 112123. DOI: 10.1016/j.jep.2019.112123 